\title{
A Three-Dimensional Tropical Cyclone Model with Parameterized Cumulus Convection
}

\author{
by \\ Masanori Yamasaki \\ Meteorological Research Institute, Tsukuba, Ibaraki 305, Japan
}

(Received July 28, 1986 ; Revised Sept. 26, 1986)

\begin{abstract}
A three-dimensional tropical cyclone model is developed with a new parameterization of cumulus convection, based on the results from the non-parameterized model of Yamasaki $(1977,83)$. In order to simulate the important features and mechanisms of tropical cyclones obtained in the non-parameterized model, cloud water and rainwater are included as predicted variables. The effects of evaporation of rainwater and convective downdrafts are taken into account. Heating due to parameterized convection is assumed to depend on the vertical velocity at a low level and the degree of the conditional instability.

Results from a numerical experiment indicate that the present model is capable of describing mesoscale convections which are pronounced in the non-parameterized model. Mesoscale convections behave in different ways, depending on the stage (or intensity) of a simulated tropical cyclone and on the location relative to the tropical cyclone center. Simulated spiral rainbands consist of mesoscale convections which form around the trailing edge of a rainband in many cases and move on the spiral band cyclonically towards the eyewall. In the case of spiral bands which are strongly affected by frictional inflow, mesoscale convections are maintained for a long period of time by successive formation of convective elements at the outer edge of the band in which warm moist air flows. The spiral bands do not behave like gravity waves. Most of the properties of mesoscale convections (including those at the pre-typhoon stage) and the tropical cyclone simulated in this study are similar to those obtained from the non-parameterized model. The essential aspects of the parameterization scheme which leads to such results and its shortcomings are also discussed.
\end{abstract}

\section{Introduction}

About fifteen years have passed since a three-dimensional tropical cyclone model first appeared and numerical studies on threedimensional tropical cyclones started (Anthes et al., 1971; Miller et al., 1972). The threedimensional model has been and will be used as a useful and necessary tool for a better understanding of asymmetric structures (e.g., Anthes, 1972) and tropical cyclone motion (e.g., Madala and Piacsek, 1975) and for numerical prediction of tropical cyclones (e.g., Ookochi, 1978). In the
Meteorological Research Institute (MRI) to which the author has belonged, development of a three-dimensional model has been made possible since a large computer became available in 1980. The ultimate goal of developing a three-dimensional model in the MRI is to improve tropical cyclone forecast. In order to do so, however, it is necessary to get a better understanding of the basic mechanisms of tropical cyclones. The present paper deals with description of a three-dimensional model which has been developed for this purpose. Some 
results obtained from numerical experiments are also presented.

The most important but difficult problem in constructing a tropical cyclone model is how the effects of moist convection are incorporated. It has been possible in recent years to deal with individual convective clouds explicitly, as done by Yamasaki $(1977,1983)$ and Willoughby et al.(1984), if an axially symmetric assumption is used. However, it is not yet appropriate at present to use this approach in the case of a three-dimensional model. As introduced by Ooyama (1964) and others, the so-called parameterization technique has to be adopted. The present study follows this approach.

Many studies have been made so far with the use of various schemes of parameterization. These have been successful in simulating important features of tropical cyclones, and the mechanisms of tropical cyclones have been fairly understood (Ooyama, 1964, 1969; Charney and Eliassen, 1964; Ogura, 1964; Yamasaki, 1968 ; Rosenthal, 1970 ; Anthes, 1972 ; Kurihara and Tuleya, 1974; Kurihara, 1975 ; Kurihara and Bender, 1982 ; Madala and Piacsek, 1975 ; Mathur, 1974, 1975; Wada, 1979 and many others). However, it has remained as an important problem to clarify to what extent the parameterization schemes used in these studies are valid for simulating tropical cyclones realistically. More realistic simulation seems to be necessary for a better understanding of the tropical cyclone.

In order to understand the interaction between moist convection and larger-scale motion and the mechanisms of larger-scale disturbances and thereby to find an appropriate parameterization scheme of moist convection, the author has made many numerical experiments with two-dimensional (axi-symmetric and slab-symmetric) models in which individual convective clouds are calculated together with a larger-scale disturbance (Yamasaki, 1975, 77a, $77 \mathrm{~b}, 79,82,83,84)$. One of the results obtained from the numerical experiments is that the model has simulated mesoscale organization of cumulus convection. It has been well known that the convective-scale downdraft and evaporative cooling (due to falling rainwater) play an important role in the formation of a new convective cloud (e.g., Byers and Braham, 1949 ; Takeda, 1971). In a numerical experiment of Yamasaki (1975), succesive formation of convective clouds under this mechanism has been simulated. In this case an interaction with larger-scale convergence, which is produced and maintained by an ensemble of clouds, plays an important role in the long lifetime of the cloud ensemble. In the revised models of Yamasaki $(1983,84)$ convective activity has a characteristic time scale ranging from a few hours to nearly ten hours, depending on the vertical shear of an environmental large-scale flow and other conditions. Such organized convection is referred to as "mesoscale convection" here, as in the previous papers. The time scale of a few hours has been known as a typical time scale of mesoscale phenomena observed in the actual atmosphere.

It has also been shown that individual mesoscale convections are further organized into a larger system. A larger-scale circulation is induced and maintained by the effects of many mesoscale convections, which in turn form successively in the larger-scale convergence field. The successive formation of mesoscale convections and the development of a larger-scale disturbance (CISK) may be classified into three types according to their mechanisms. (1) The downdraft and evaporative cooling associated with falling rainwater play an essential role in the formation of mesoscale convections. In addition, in order that mesoscale convections may form successively for a long time, a larger-scale lowlevel inflow of warm moist air is one of the necessary conditions. Mesoscale convections and a larger-scale circulation interact in a cooperative way. Surface friction is not important. Although the Coriolis force is important to intensification of rotational winds (and vorticity), it is not essential to convective activities. This type of CISK was studied in Yamasaki $(1975,77 b, 79,84)$. (2) The downdraft and evaporative cooling are still essential. However, frictional inflow plays some role in the formation of mesoscale convections. That is, convective activities are enhanced by frictional inflow. Another role of frictional inflow is to prevent major convective activities from 
expanding over a larger area. Roughly speaking, this type of CISK becomes pronounced when the wind velocity attains about $10 \mathrm{~ms}^{-1}$. Since surface friction is important the Coriolis force is also important. This CISK was studied in Yamasaki (1977a, 83). It should be remarked that individual convective activities are not directly controlled by frictional "convergence" (although frictional convergence contributes to convective activities as a whole). In this sense this CISK is different from the original CISK (Ooyama, 1964 ; Charney and Eliassen, 1964) in which Ekman pumping is an important mechanism controlling convective activities. (3) Convective activities are strongly controlled by frictional convergence, as emphasized in the original CISK theory. The eyewall circulation is the most typical example. The properties of the eyewall circulation were studied in Yamasaki (1977a, 83).

It should be remarked that these three mechanisms are continuous in the intensification process of weak tropical disturbances and tropical cyclones. It should also be mentioned that it is possible for the three different mechanisms to appear simultaneously; that is, convective activities in different areas in a tropical cyclone may be due to different mechanisms.

As is clear from the results mentioned above, the eyewall circulation can be essentially simulated by existing parameterization schemes (although efforts will still be needed to improve it). However, as for other aspects of tropical cyclones, particularly at the formation and the early development stage, it appears that existing tropical cyclone models have much to be improved. Spiral rainbands may be better simulated (even at the mature stage) if we use a more appropriate model. It was suggested by Anthes (1972) and Kurihara and Tuleya (1974) that spiral rainbands might be internal gravity waves modified by convective heating. In the non-parameterized model of Yamasaki (1977a, 83), rainbands did not behave like gravity waves but were obtained as organization of many mesoscale convections in which the downdraft and evaporative cooling played an essential role. Needless to say, the validity of the results obtained from the non- parameterized model has to be clarified from other modeling and observational studies in the future. However, the present study is based on the results from the non-parameterized model. (A support of the studies with the non-parameterized model was given by Ooyama (1982).) The primary objectives of this study are to improve cumulus parameterization and to find how it works in a "three-dimensional" model. (The essence of this study was presented at the meeting of the Meteorological Society of Japan in 1984, and an earlier result from an "axisymmetric" model was presented in 1981.)

\section{Thermodynamical aspect of the model}

2.1 basis for cumulus parameterization

One of the important points in developing a parameterization scheme is what to parameterize and what to describe explicitly. When we use a grid size of $10 \mathrm{~km}$ or $20 \mathrm{~km}$ in a numerical model, the eyewall and rainbands can be described explicitly. When we use a larger grid size such as $100 \mathrm{~km}$, the rainbands have to be parameterized. The latter problem seems to be more difficult than the former. This is left for future studies. The primary objective of this study is to find a parameterization scheme which may be appropriate for the former problem.

It is assumed in this study that the rainband is due to successive formation of such mesoscale convections as defined above. It is intended that cumulus convection is parameterized and that mesoscale convection is explicitly resolved. In a model of Yamasaki (1983), a typical time scale of mesoscale convection is a few hours at the stage when frictional inflow is not very significant. The horizontal scale of the ascending motion of mesoscale convection (in terms of the width in the case of roll-like convection) is a few tens of kilometers. In order to describe mesoscale convection with this scale properly, a horizontal grid size of $5 \mathrm{~km}$ or less is desirable. The scale of mesoscale convection becomes larger, depending on the large--scale conditions. In particular, when frictional flow becomes very significant, the time and horizontal scales of convective activities become much larger, although the time scale of cumulus convection 
is still pronounced (Yamasaki, 1983). In this case larger grid sizes such as $20 \mathrm{~km}$ or more may be justified. As suggested from the properties of mesoscale convection mentioned above, the scale separation between cumulus convection and mesoscale convection is not large, and therefore parameterizability may be somewhat doubtful, particularly when frictional flow is weak. This problem remains to be studied. Nevertheless, it is necessary to construct a parameterized model until it becomes possible for cumulus convection to be resolved explicitly.

The most important aspect of the parameterization scheme proposed in this paper is that the effects of cooling due to evaporation of rainwater and downdrafts associated with cumulus convection and mesoscale convection are taken into account. In addition, since the properties of mesoscale convection are greatly modified by such factors as frictional flow and vertical shear, it is important to find a parameterization scheme which is acceptable for a wide range of large-scale conditions. An intention of this study is to formulate the mechanisms which occur in a tropical cyclone model of Yamasaki $(1983)^{*}$, and thereby to find a parameterization scheme by which tropical cyclones can be simulated more realistically.

\section{2 basic equations}

For the reasons mentioned above, the mixing ratios of cloud water and rainwater as well as that of water vapor are taken to be predicted variables. Basic equations used are similar to those derived by Ooyama (1971), Yanai et al. (1973) and Arakawa and Schubert (1974) in that cloud mass flux $M^{c}$ and detrainment $D_{e t}$ are used.

$$
\begin{aligned}
\frac{d \theta}{d t}= & \frac{M^{c}}{\rho} \frac{\partial \theta}{\partial z}+D_{e t}\left(\theta^{c}-\theta\right)+D_{W}(\theta) \\
& +\frac{1}{C_{p} \pi}\left(Q_{\mathrm{COND}}-Q_{\mathrm{EVP}}-Q_{\mathrm{RAD}}\right)+F(\theta) \\
\frac{d q_{v}}{d t}= & \frac{M^{c}}{\rho} \frac{\partial q_{v}}{\partial z}+D_{e t}\left(q_{v}^{c}-q_{v}\right)+D_{W}\left(q_{v}\right) \\
& -q_{\mathrm{COND}}+q_{\mathrm{EVP}}+F\left(q_{v}\right)
\end{aligned}
$$

$$
\begin{aligned}
\frac{d q_{c}}{d t}= & \frac{M^{c}}{\rho} \frac{\partial q_{c}}{\partial z}+D_{e t}\left(q_{c}^{c}-q_{c}\right)+D_{W}\left(q_{c}\right) \\
& +q_{\mathrm{COND}}-q_{\mathrm{CONV}}+F\left(q_{c}\right) \\
\frac{d q_{r}}{d t}= & \frac{M^{c}}{\rho} \frac{\partial q_{r}}{\partial z}+D_{e t}\left(q_{r}^{c}-q_{r}\right)+\delta q_{r} \\
& -q_{\mathrm{EVP}}+q_{\mathrm{CONV}}+V_{T} \frac{\partial q_{r}}{\partial z}
\end{aligned}
$$

where $\theta$ is the potential temperature, $q_{v}$ the mixing ratio of water vapor, $q_{c}$ mixing ratio of cloud water and $q_{r}$ mixing ratio of rainwater. These quantities indicate the dependent variables which are resolved by the grid used (referred to as grid-scale or grid-resolved variable). The values for the cloud are indicated by $\theta^{c}, q_{v}^{c}, q_{c}^{c}$ and $q_{r}^{c}$. The effects of implicit convection are represented by terms including $M^{c}$ cloud mass flux, $D_{e t}$ detrainment, $D_{W}$ downdraft effects and $\delta q_{r}$ the rate of rainwater increase due to fall of $q_{r}^{c}$ (rainwater inside the cloud) into the subcloud layer. As for other notations, $Q_{\text {cond }}$ indicates heating due to grid-scale condensation of water vapor (evaporation of cloud water is indicated by a negative value), $Q_{\mathrm{EVP}}$ cooling due to evaporation of rainwater, $q_{\mathrm{COND}}$ and $q_{\mathrm{EVP}}$ changes of the mixing ratios due to condensation and evaporation of rainwater, respectively, $q_{\mathrm{coNv}}$ conversion rate (due to autoconversion and collection) from cloud water to rainwater, $V_{\mathrm{T}}$ fall velocity of rainwater relative to the air, $Q_{\mathrm{RAD}}$ radiative cooling, $F$ eddy diffusion effects, $\rho$ density, $z$ height, $C_{p}$ specific heat at constant pressure and $\pi$ Exner function defined by $\left(p / p_{00}\right)^{R / C_{p}}, p$ pressure, $p_{00}$ reference pressure $(1,000 \mathrm{mb})$ and $R$ gas constant.

\section{3 grid-resolved effects}

The grid-resolved condensation (or evaporation of cloud water) $Q_{\text {Cond }}$ is determined so that supersaturation may not take place. The saturation mixing ratio $q_{v s}$ is approximately given by

$$
\begin{aligned}
q_{v s} & =\left(a_{2} T^{2}+a_{1} T+a_{0}\right)\left(1-p^{\prime}+p^{\prime 2}\right) \\
p^{\prime} & \equiv\left(p-p_{\mathrm{B}}\right) / p_{\mathrm{B}}
\end{aligned}
$$

where $T$ is the temperature, and $a_{2}, a_{1}$ and $a_{0}$ are functions of $p_{\mathrm{B}}$ (basic state pressure) and $T_{\mathrm{B}}$ (basic state temperature), which are approximately determined from Teten's formula, as done in $\mathrm{Y}$. The evaporation of rainwater is

\footnotetext{
* Referred to as $\mathrm{Y}$ in this paper.
} 
assumed to be

$$
q_{\mathrm{EVP}}=4.32\left(q_{v s}-q_{v}\right)\left(\rho q_{r}\right)^{0.65}
$$

The following relations hold:

$$
\begin{aligned}
Q_{\mathrm{COND}} & =L q_{\mathrm{COND}} \\
Q_{\mathrm{EVP}} & =L q_{\mathrm{EVP}}
\end{aligned}
$$

where $L$ is the latent heat of condensation. The conversion from cloud water to rainwater consists of autoconversion $A_{\text {conv }}$ and collection $C_{o l}$ :

$$
\begin{aligned}
q_{\mathrm{CONV}} & =A_{\mathrm{CONV}}+C_{o l} \\
A_{\mathrm{CONV}} & =A_{1}\left(q_{c}-A_{2}\right)\left(q_{c}>A_{2}\right) \\
C_{o l} & =929 q_{c}\left(\rho q_{r}\right)^{0.875}
\end{aligned}
$$

where we take $\mathrm{A}_{1}=10^{-3} \mathrm{~s}^{-1}\left(\mathrm{~A}_{1}=0\right.$ for $\left.q_{c} \leq A_{2}\right)$ and $A_{2}=0.5 \mathrm{~g} \mathrm{~kg}^{-1}$. The fall velocity $\left(\mathrm{m} \mathrm{s}^{-1}\right)$ of rainwater relative to the air is assumed to be

$$
V_{\mathrm{T}}=29\left(\rho q_{r}\right)^{0.125}
$$

These assumptions concerning $q_{\mathrm{EVP}}, A_{\mathrm{CoNv}}, C_{o l}$ and $V_{\mathrm{r}}$ are based on parameterization schemes for cloud physical processes proposed by Kessler(1969) and used in many cloud modeling studies. It is assumed that these parameterizations also hold for non-convective clouds. This is a very crude assumption and should be revised in the future.

\section{4 cumulus parameterization}

In the following we make assumptions on quantities concerning the cloud; $M^{c}, D_{e t}, \theta^{c}$, $q_{v}{ }^{c}, q_{c}{ }^{c}, q_{r}{ }^{c}, D_{W}$ and $\delta q_{r}$. The cloud mass flux $M^{c}$, which is the most important quantity in the parameterization scheme, is assumed to be proportional to $w^{*}$, the vertical velocity at some level $z=z^{*}$, if $w^{*}$ is positive, where $z^{*}$ is a height which is somewhat lower than the cloud base height. For simplicity, $z^{*}$ is assumed to be constant. The assumption for $M^{c}$ may be written as follows :

$$
\begin{aligned}
& \frac{M^{c}}{\rho}=\left\{\begin{array}{cc}
Y w^{*} & \left(w^{*}>0\right) \\
0 & \left(w^{*} \leq 0\right)
\end{array}\right. \\
& Y \equiv Y^{*} \eta
\end{aligned}
$$

where $\eta$ is the normalized cloud mass flux which is taken to be unity at $z^{*}$, and $Y^{*}$ is a value of $Y$ at $z^{*}$; in other words, the ratio of the cloud mass flux $M^{c}$ to the grid-scale mass flux $\rho w$ at $z^{*}$. Although the assumption (10) is similar to that proposed by Ooyama(1964) and used in numerical experiments (e.g., Yamasaki, 1968), the intention of using this assumption is different in that mesoscale features are to be described.

It is assumed that the cloud mass flux $M^{c}$ or $\eta$ can be determined from the properties of clouds of a single type. The coexistence of various types of clouds is not taken into account for a given large-scale condition. Another assumption is that the cloud mass flux strongly depends on the cloud buoyancy $B$. In the present study, $\eta$ is assumed to be

$$
\eta=1+C_{\eta} B
$$

where $C_{\eta}$ is a parameter and $B$ is a measure of the cloud buoyancy (or the degree of the conditional instability) defined by

$$
\begin{aligned}
B \equiv & T^{c}\left(1+0.608 q_{v}{ }^{c}-q_{c}{ }^{c}\right) \\
& -T\left(1+0.608 q_{v}-q_{c}\right)
\end{aligned}
$$

where $T^{c}$ and $T$ are the temperatures of the cloud and the grid-scale fields, respectively. (In this study the drag of rainwater is not included in determining $B$.) The assumption (12) is also different from that of Arakawa and Schubert (1974) in which the normalized cloud mass flux was determined by turbulent entrainment. Validity of (12) has not been clarified. However, (12) is similar to a parameterization scheme used in Yamasaki (1968) in that heating due to parameterized convection is assumed to depend on the difference between $T^{c}$ and $T$. This property is similar to that proposed by Kuo (1965), although physical basis for its use in the present study is different. According to the results obtained from numerical experiments of tropical cyclones (Yamasaki, 1968; Rosenthal, 1970) and from numerical experiments with explicit calculation of clouds (Yamasaki, 1979, 1983), the assumption (12) seems to be appropriate as a first approximation. As for parameter $C_{\eta}$, it is assumed to be 0.2 for $B>0$ in the layers well above $z^{*}$. Since $\eta$ is unity at $z^{*}$ by definition, $C_{\eta}$ is assumed to be zero at $z^{*}$. When $B$ is negative, $\mathrm{C}_{\eta}$ is assumed to be 1.0 . This value is chosen so that $\eta$ may become zero for $B=-1$. Positive values of $\eta$ for $-1<B<$ 0 correspond to overshooting of clouds into layers of negative buoyancy.

An important but difficult problem is how $Y^{*}$ should be determined. It is assumed that $Y^{*}$ is determined by the degree of conditional instability at lower levels of the cloud layer. A 
possible assumption is

$$
Y^{*}=\left\{\begin{array}{lr}
1+C^{* *}\left(\eta^{* *}-1\right) & \left(\eta^{* *}>1\right) \\
\eta^{* *} & \left(1 \geq \eta^{* *} \geq 0\right)
\end{array}\right.
$$

where $C^{* *}$ is a parameter and $\eta^{* *}$ is the value of $\eta$ averaged for a layer or at a level $z^{* *}$ which is $1 \mathrm{~km}$ or a few kilometers higher than $z^{*}$. The assumption (14) is taken so that $Y^{*}$ may become 1 when the stratification in the lower cloud layer becomes moist neutral $\left(\eta^{* *} \rightarrow 1\right)$. According to (12), $\eta$ is 1 when $B$ is zero. These conditions are required in order that explicitly resolved convection may not grow when the stratification becomes neutral. The importance of $\eta$ in the lower cloud layer as well as $Y^{*}$ was shown by linear theory (Syono and Yamasaki, 1966). The specification of a somewhat arbitrary parameter $C^{* *}$ will be given later.

The cloud properties $T^{c}, q_{v}{ }^{c}$ and $q_{c}{ }^{c}$ can be determined from four conditions: (1) balance of moist static energy, (2) balance of the sum of water vapor and cloud water, (3) saturation condition and (4) cloud base condition, as done by Yanai et al. (1973) and Arakawa and Schubert (1974). Before describing these conditions, some definitions and assumptions have to be given. In this paper moist static energy is defined by

$$
h=C_{p} T_{h}+g z+L q_{v}
$$

where $g$ is the acceleration of gravity and $T_{h}$ is the air temperature obtained by changing the pressure $p$ adiabatically to the basic state pressure $p_{\mathrm{B}}$; that is,

$$
T_{h}=T\left(p_{\mathrm{B}} / p\right)^{R / c_{p}}
$$

It should be remarked that it is not appropriate to use $T$ instead of $T_{h}$. This is because the pressure fall is very large in the case of tropical cyclones. As is well known, lower pressure with the same temperature means higher equivalent potential temperature. It is important to take account of this effect. (For consistency, similar modification has to be made for the third term in (15) in view of the well-known conservation of $C_{p} T+L q_{v}$. The use of (15) introduces some difference in the determination of cloud temperature $T^{c}$. However, this is probably very small. It is important that the use of (15) gives a much more appropriate determination of $T^{c}$ than the usual definition of $h$. In future studies, however, the use of equivalent potential temperature may be desirable.)

Another assumption is that turbulent entrainment $E_{T}$ and detrainment $D_{T}$ are taken into account in modeling the cloud. Using finite difference representation, we assume

$$
\left(E_{T}\right)_{l}=E_{D} \eta_{l+1}(\Delta z)_{l}
$$

where $E_{D}$ is an entrainment parameter, suffix $l$ indicates a level in the numerical model (downward increasing), $(\Delta z)_{\iota}$ is the depth of the cloud layer under consideration, and $\eta_{l}$ and $\eta_{l+1}$ are the values of $\eta$ at the top and the base of the layer, respectively. By an assumption, $D_{T}$ is equal to $E_{T}$ :

$$
D_{T}=E_{T}
$$

In addition to turbulent entrainment and detrainment, there exists dynamic entrainment or detrainment, depending on the sign of $\left(\eta_{l}\right.$ $\left.-\eta_{l+1}\right)$.

The assumption that the moist static energy is nearly balanced for implicit convection may be written as follows:

$$
\begin{aligned}
& -\eta_{l} h_{l}^{c}+\eta_{l+1} h_{l+1}{ }^{c}+\left(\eta_{l}-\eta_{l+1}\right) \widetilde{h}_{l} \\
& -\left(D_{T}\right)_{l} \frac{h_{l}^{c}+h_{l+1}{ }^{c}}{2}+\left(E_{T}\right)_{l} \frac{h_{l}+h_{l+1}}{2}=0
\end{aligned}
$$

where

$$
\widetilde{h}_{l} \equiv \begin{cases}\frac{h_{l}+h_{l+1}}{2} & \left(\eta_{l}-\eta_{l+1}>0\right) \\ \frac{h_{l}^{c}+h_{l+1}{ }^{c}}{2} & \left(\eta_{l}-\eta_{l+1}<0\right) .\end{cases}
$$

In obtaining the above relation,, moist static energy outside the cloud is approximated by the grid-scale moist static energy $h$. Eq. (19) is used to determine $h_{l}{ }^{c}$ if $h_{l+1}{ }^{c}, \eta_{l+1}$ and $\eta_{l}$ are known.

The budget of the sum of water vapor and cloud water is

$$
\begin{aligned}
& -\eta_{l} Q_{l}{ }^{c}+\eta_{l+1} Q_{l+1}{ }^{c}+\left(\eta_{l}-\eta_{l+1}\right) \widetilde{Q}_{l} \\
& -\left(D_{T}\right)_{l} \frac{Q_{l}{ }^{c}+Q_{l+1}{ }^{c}}{2}+\left(E_{T}\right)_{l} \frac{Q_{l}+Q_{l+1}}{2} \\
& -C_{\mathrm{CONV}}(\Delta z)_{l} \frac{\eta_{l}+\eta_{l+1}}{2} \frac{\left(q_{c}^{c}\right)_{l}+\left(q_{c}{ }^{c}\right)_{l+1}}{2}=0
\end{aligned}
$$

where

$$
Q \equiv q_{v}+q_{c}
$$

is the sum of water vapor and cloud water. The 
meaning of $\widetilde{Q}_{l}$ is similar to $\widetilde{h}_{l}$. The effect of conversion from cloud water to rainwater (due to autoconversion and collection) is taken into account following Arakawa and Schubert (1974). The coefficient $C_{\text {conv }}$ concerning the conversion is taken to be $2 \times 10^{-3} \mathrm{~m}^{-1}$, although it may be more realistic to assume that $C_{\mathrm{CoNv}}$ depends on the vertical velocity in the cloud. This value is chosen so that the realistic profiles of $q_{c}^{c}$ and $q_{r}^{c}$ may be obtained. Relation (20) is used to determine $\left(q_{c}^{c}\right)_{l}$ if other quantities are known.

The condition that it is saturated inside the cloud may be written as follows :

$$
q_{v}^{c}=\left[a_{2}\left(T^{c}\right)^{2}+a_{1}\left(T^{c}\right)+a_{0}\right]\left(1-p^{\prime}+p^{\prime 2}\right)
$$

where $p^{\prime}$ is defined by (6). Moist static energy in the cloud is given by

$$
h^{c}=C_{p} T^{c}\left(p_{B} / p\right)^{R / C_{p}}+g z+L q_{v}^{c}
$$

Relations (21) and (22) hold for each level $l$. From (21) and (22), $T^{c}$ and $q_{v}^{c}$ can be determined if $h^{c}$ is known from (19).

The conditions at the base of the cloud updraft, which is taken to be $z^{*}$, are assumed to be as follows:

$$
\begin{aligned}
& h_{l^{*}}^{c}=\left(h_{l^{*}}+h_{l^{*}+1}\right) / 2 \\
& \left(q_{v}^{c}\right)_{l^{*}}=\left[\left(q_{v}\right)_{l^{*}}+\left(q_{v}\right)_{l^{*}+1}\right] / 2 \\
& \left(q_{c}^{c}\right)_{l^{*}}=\left[\left(q_{c}\right)_{l^{*}}+\left(q_{c}\right) l^{*+1}\right] / 2
\end{aligned}
$$

where $l^{*}$ is a level corresponding to $z^{*}$. The three right-hand terms may be replaced by $h_{l}$, $\left(q_{v}\right)_{l}$ and $\left(q_{c}\right)_{l^{*}}$, respectively. In this study, it is assumed that the cloud properties at $z^{*}$ are different from those of the grid-scale fields, and that these are determined by the grid-scale fields at somewhat lower levels. Another condition at $z^{*}$ is, by definition,

$$
\eta_{l^{*}}=1
$$

Four relations (19), (20), (21) and (22) can be solved to obtain $h^{c}, T^{c}$ (and $\theta^{c}$ ), $q_{v}^{c}$ and $q_{c}^{c}$ by the use of lower boundary conditions (23) and (24), if $\eta$ is known. However, the value of $\eta$ is related to $T^{c}, q_{v}{ }^{c}$, and $q_{c}{ }^{c}$ through (12) and (13). The values of $\eta_{l}, h_{l}^{c}, T_{l}^{c}, \quad\left(q_{v}^{c}\right)_{l}$ can be determined iteratively when the values at level $(l+1)$ are known. However, an approximate solution can be obtained by the following method. We define $\varepsilon$ by $\varepsilon \equiv \eta_{l}-\eta_{l+1}$. First, $B_{l}$ is obtained from (13) by assuming $\varepsilon=0$; that is, $\eta_{l}=\eta_{l+1}$. The new value of $\eta_{l}$ is obtained from (12). The value of $\varepsilon$ for this value of $\eta_{l}$ is denoted by $\varepsilon_{1}$. As the next step, a new value of $\eta_{l}$ is obtained by assuming $\varepsilon=\varepsilon_{1}\left(\eta_{l}=\eta_{l+1}+\varepsilon_{1}\right)$. The new value of $\varepsilon$ is denoted by $\varepsilon_{2}$. An approximate solution $\varepsilon_{0}$ can be obtained by assuming that a linear relation holds for an assumed $\varepsilon$ and a calculated $\varepsilon$; that is,

$$
\begin{aligned}
& \varepsilon_{0}=\varepsilon_{1}{ }^{2} /\left(2 \varepsilon_{1}-\varepsilon_{2}\right) \\
& \eta_{l}=\eta_{l+1}+\varepsilon_{0}
\end{aligned}
$$

This relation holds for both positive and negative values of $\varepsilon$. If $\eta_{l}$ is negative, $\eta_{l}$ is set to zero. Thus the level of the cloud top can be determined.

The rainwater in the cloud $q_{r}^{c}$ is treated in a way different from $q_{v}{ }^{c}$ and $q_{c}{ }^{c}$. As described before, $q_{v}^{c}$ and $q_{c}^{c}$ are diagnostically determined. On the other hand, $q_{r}{ }^{c}$ is determined prognostically. This is because $q_{r}^{c}$ can not be determined only from cloud properties at an instanteneous time. It is also important to note that the rainwater mixing ratio averaged for the whole area including cloud-free area is necessary to determine $\delta q_{r}$, which is the conversion rate from $q_{r}^{c}$ to $q_{r}$ in eq. (4). When the area-averaged mixing ratio is denoted by $Q r^{c}$ and the cloud fraction by $a^{c}$, the following relation holds :

$$
Q_{r}{ }^{c}=a^{c} q_{r}{ }^{c} \text {. }
$$

An equation for $Q_{r}^{c}$ may be written as follows :

$$
\frac{d Q_{r}{ }^{c}}{d t}=a^{c} V_{T}{ }^{c} \frac{\partial q_{r}{ }^{c}}{\partial z}+\delta Q_{r}{ }^{c}-\delta q_{r}
$$

where $V_{T}^{c}$ is the fall velocity of rainwater relative to the air and $a^{c} q_{r}^{c}$ can be replaced by $Q_{r}{ }^{c}$. The assumption for $V_{T}^{c}$ is similar to $V_{T}$ given by $(9)$; that is,

$$
V_{T}^{c}=29\left(\rho q_{r}^{c}\right)^{0.125}
$$

Since $a^{c}$ is not known in the present model, $Q_{r}^{c}$ is used instead of $q_{r}^{c}$ in determining $V_{T}^{c}$. This approximation does not seem to be seriously inappropriate because $V_{T}^{c}$ does not strongly depend on the mixing ratio. This will be improved in the future, however. As for the vertical velocity of the air included in the time derivative in (25), it is desirable to use the vertical velocity inside the cloud $w^{c}$. Although we have a relation $M^{c}=\rho a^{c} w^{c}$, this relation includes unknown quantity $a^{c}$. In this study it 
may be assumed that $w^{c}$ depends on $w$ as follows :

$$
w^{c}=C_{w} w
$$

where $C_{w}$ is assumed to also depend on $w$. For large values of $w, C_{w}$ is taken to be unity, and for small values of $w$, it ranges from 1 to some value such as 5 . It is also assumed that the rainwater is advected horizontally by the gridresolved horizontal wind.

The second term $\delta Q r^{c}$ in (25) indicates the generation of rainwater due to conversion from cloud water. This is given by

$$
\delta Q_{r}{ }^{c}=C_{\mathrm{CONv}} q_{c}{ }^{c} \eta Y^{*} w^{*}\left(w^{*}>0\right)
$$

where $C_{\text {conv }}$ is the conversion coefficient which appears in (20). The third term $\delta q_{r}$ is assumed to be

$$
\delta q_{r}=C_{R} Q_{r}^{c}
$$

where $C_{R}$ is taken to be $10^{-3} \mathrm{sec}^{-1}$ in a layer of $z^{*}$ and infinitely large well below it. The latter means that all $Q_{r}^{c}$ is treated as $q_{r}$ in the subcloud layer.

The detrainment $D_{e t}$ in (1)-(4) may be expressed as the sum of the dynamic detrainment and turbulent detrainment as follows:

$$
D_{e t}=\left\{\begin{array}{lr}
-\frac{\partial \eta}{\partial z} Y^{*} w^{*}+E_{D} \eta Y^{*} w^{*} & (\partial \eta / \partial z<0) \\
E_{D} \eta Y^{*} w^{*} & (\partial \eta / \partial z \geq 0)
\end{array}\right.
$$

where $E_{D}$, which appears in (17), is used because of an assumption of (18). The detrainment term in rainwater equation (4) is not included in this study partly because this effect is small.

The effect of the downdraft associated with parameterized convection is formulated in a form similar to that of the vertical eddy diffusion term.

$$
D_{W}(S)=\frac{1}{\rho} \frac{\partial}{\partial z}\left(K_{c} \frac{\partial S}{\partial z}\right)
$$

where $S$ may be $\theta, q_{v}$ or $q_{c}$, and $K_{c}$ is the coefficient concerning convective downdraft $\left(\mathrm{K}_{c}=0\right.$ above $\left.z^{*}\right)$. It is a difficult problem how to determine $K_{c}$. As a first step, it is assumed that $K_{c}$ is proportional to $w$ and $\left(Q_{r}{ }^{c}\right)^{1 / 2}$ at $z^{*}$; that is,

$$
K_{c}=K_{D} \frac{w^{*}}{w_{0}}\left(\frac{Q_{r}{ }^{c *}}{Q_{r 0}{ }^{c}}\right)^{1 / 2} \quad\left(w^{*}>0\right)
$$

where $K_{D}$ is a parameter, and $w_{0}=1 \mathrm{~m} \mathrm{~s}^{-1}$ and
$Q_{r 0}{ }^{c}=1 \mathrm{~g} \mathrm{~kg}^{-1}$. The value of $K_{D}$ indicates the value of $K_{c}$ in the case of $w^{*}=1 \mathrm{~m} \mathrm{sec}^{-1}$ and $Q_{r}{ }^{* *}=1 \mathrm{~g} \mathrm{~kg}^{-1}$.

\section{5 eddy diffusion}

The eddy diffusion terms in (1)-(3) are assumed to be

$$
\begin{aligned}
& F(S)=F_{H}(S)+F_{V}(S) \\
& F_{H}(S)=K_{H}\left(\frac{\partial^{2} S}{\partial x^{2}}+\frac{\partial^{2} S}{\partial y^{2}}\right) \\
& F_{V}(S)=\frac{1}{\rho} \frac{\partial F_{S}}{\partial z} \\
& F_{S}=K_{V} \frac{\partial S}{\partial z}
\end{aligned}
$$

where $K_{H}$ and $K_{V}$ indicate the horizontal and vertical eddy diffusion coefficients, respectively.

At the sea surface $(z=0), F_{S}$ is assumed to be

$$
\begin{aligned}
& F_{\theta}=\rho C_{E}|V|\left(T_{\text {sea }}-T_{a i r}\right) / \pi \\
& F_{q_{v}}=\rho C_{E}|V|\left\{\left(q_{v}\right)_{\text {sea }}-\left(q_{v}\right)_{a i r}\right\}
\end{aligned}
$$

where $C_{E}$ is a coefficient, $|V|$ the wind velocity, $T_{\text {sea }}$ surface temperature, $T_{\text {air }}$ the temperature of the surface air and $\left(q_{v}\right)_{a i r}$ mixing ratio of water vapor of the surface air. The saturation mixing ratio corresponding to $T_{\text {sea }}$ is given by

$$
\stackrel{\left(q_{v}\right)_{\text {sea }}}{\quad=\left[a_{2} T_{\text {sea } a}^{2}+a_{1} T_{\text {sea }}+a_{0}\right]\left(1-p_{\text {séa }}^{\prime}+p_{\text {sea }}^{\prime 2}\right)}
$$

where $p_{S e^{\prime} a}$ is the surface value of the pressure perturbation defined by (6).

\section{Dynamical aspect of the model}

In the model developed in this study, sigma is used as the vertical coordinate, where it is defined by

$$
\sigma=\frac{p-p_{T}}{p_{S}-p_{T}}
$$

where $p_{s}$ is the surface pressure, and $p_{T}$ is the pressure of the top of the atmosphere under consideration. The thermodynamic equations described in the previous section may be rewritten using relations

$$
\begin{aligned}
& \frac{\partial}{\partial z}=-\frac{\rho g}{p_{S}-p_{T}} \frac{\partial}{\partial \sigma} \\
& \frac{M^{c}}{\rho} \frac{\partial}{\partial z}=Y W^{*} \frac{\partial}{\partial \sigma} \\
& \frac{d}{d t}=\frac{\partial}{\partial t}+u \frac{\partial}{\partial x}+v \frac{\partial}{\partial y}+W \frac{\partial}{\partial \sigma}
\end{aligned}
$$


where $x$ and $y$ are the horizontal coordinates directed eastward and northward, $u$ and $v$ are $x$-and $y$-components of the horizontal velocity, respectively. The vertical $\sigma$-velocity is denoted by $W(\equiv d \sigma / d t)$. The equations of motion in $x$ and $y$-directions may be written as follows:

$$
\begin{aligned}
& \frac{d u}{d t}-f v=-\frac{\partial \phi}{\partial x}-Z \frac{\partial p_{s}}{\partial x}+F(u) \\
& \frac{d v}{d t}+f u=-\frac{\partial \phi}{\partial y}-Z \frac{\partial p_{s}}{\partial y}+F(v)
\end{aligned}
$$

where

$$
Z \equiv \sigma R T / p,
$$

$\phi$ the geopotential and $f$ the Coriolis parameter. We use $\beta$-plane approximation

$$
f=f_{0}+\beta y
$$

where $f_{0}$ is the value of the Coriolis parameter at $y=0$, and $\beta$ is the latitudinal variation of the Coriolis parameter, which is taken to be $2 \times$ $10^{-11} \mathrm{sec}^{-1} \mathrm{~m}^{-1} . F$ is the eddy viscosity which takes the same form as that for the thermodynamic variables except for the surface; that is,

$$
\begin{aligned}
& F(u)=F_{H}(u)+F_{V}(u) \\
& F_{H}(u)=K_{H}\left(\frac{\partial^{2} u}{\partial x^{2}}+\frac{\partial^{2} u}{\partial y^{2}}\right) \\
& F_{V}(u)=\frac{1}{\rho} \frac{\partial \tau_{x}}{\partial z} \\
& \tau_{x}= \begin{cases}K_{V} \frac{\partial u}{\partial z} & (z>0) \\
\rho C_{D}|V| u & (z=0)\end{cases}
\end{aligned}
$$

where $C_{D}$ is the drag coefficient and $\partial / \partial z$ is replaced by $\partial / \partial \sigma$ using (37). The same form as (40) holds for $v$.

The equation of continuity and the hydrostatic equation are

$$
\begin{aligned}
& \frac{\partial p_{s}}{\partial t}+\frac{\partial\left(p_{s} u\right)}{\partial x}+\frac{\partial\left(p_{s} v\right)}{\partial y}+\frac{\partial\left(p_{s} W\right)}{\partial \sigma}=0 \\
& \frac{\partial \phi}{\partial \sigma}=-\frac{R T}{p}\left(p_{S}-p_{T}\right)
\end{aligned}
$$

The upper and lower boundary conditions are

$$
\begin{aligned}
& W=\frac{\partial u}{\partial \sigma}=\frac{\partial v}{\partial \sigma}=0 \quad \text { at } \sigma=0 \\
& W=0 \quad \text { at } \sigma=1
\end{aligned}
$$

The boundary condition for $W$ determines $p_{s}$ as follows:

$$
\frac{\partial p_{s}}{\partial t}=-\int_{0}^{1}\left\{\frac{\partial\left(p_{s} u\right)}{\partial x}+\frac{\partial\left(p_{s} v\right)}{\partial y}\right\} d \sigma
$$

Using (41) and (44), $W$ can be determined. The orography is not taken into account. In this case

$$
\phi=0 \quad \text { at } \sigma=1
$$

The geopotential at each level $\phi$ is determined from (42) and (45).

\section{Numerical model}

The vertical structure of the numerical model used in this study is shown in Fig. 1. The model has five layers where horizontal velocities $u_{\imath}$ and $v_{\imath}(l=1,2, \cdots, 5)$ are defined. Although the number of the layers $L$ is an imput parameter which can be easily changed in the computer program, numerical experiments presented in this study have been performed with $L=5$. The geopotential $\phi_{l}$ is predicted at the same level as $u_{\imath}$ except that $\phi_{6}(=0)$ is defined at the surface $\left(\sigma_{6}=1\right)$. The equations of motion are applied at five layers. The thermodynamic variables $S\left(\theta, q_{v}, q_{c}, q_{r}\right.$, $\left.Q_{r}{ }^{c}\right)$ are defined at levels different from $\phi$. These thermodynamic variables may be considered as the average of the layers indicated by the solid lines with arrows in Fig. 1 . The

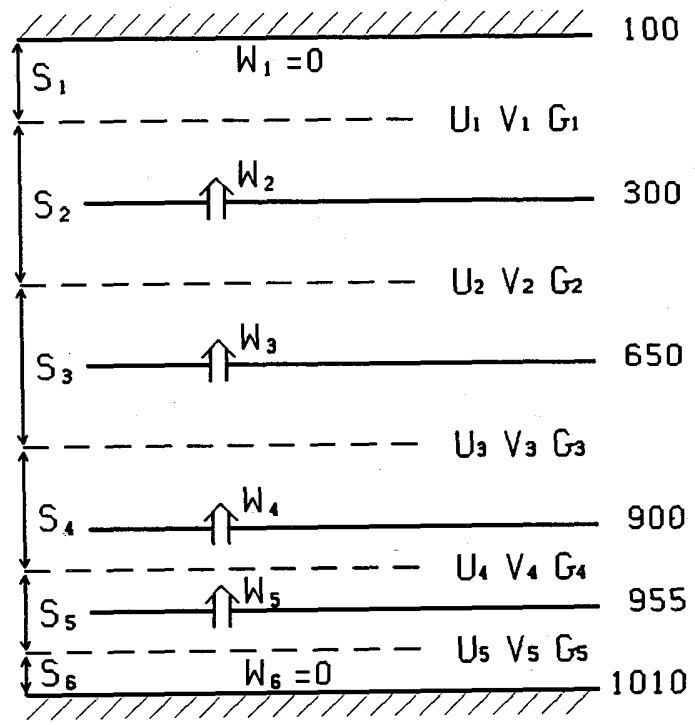

Fig. 1 Vertical structure of the $\sigma$-coordinate fivelayer model used in this study. In the figure, $\mathrm{G}$ indicates $\phi$, and $S$ indicates $\theta, q_{v}$, $q_{c}$ and $q_{r}$. Numerals in the right part indicate basic state pressures $(\mathrm{mb})$ at each level. Corresponding values of $\sigma$ are given in Table 1. 
Table 1 Basic state used in this study.

\begin{tabular}{|c|c|c|c|c|c|c|}
\hline \multicolumn{3}{|c|}{ assumed parameters } & & \multirow{2}{*}{$\underset{(\mathrm{km})}{z}$} & \multirow{2}{*}{$\begin{array}{c}\rho_{B} \\
\left(\mathrm{~kg} / \mathrm{m}^{3}\right)\end{array}$} & \multirow{2}{*}{$\begin{array}{c}\left(R_{H}\right)_{B} \\
(\%)\end{array}$} \\
\hline$p_{B}$ & $T_{B}$ & $\left(q_{v}\right)_{B}$ & & & & \\
\hline 100 & 200.0 & $(0.02)$ & 0.000 & 15.7 & 0.17 & $(-$ \\
\hline 300 & 241.0 & 0.5 & 0.2 & 9.3 & 0.4 & 60 \\
\hline 650 & 277.2 & 6.2 & 0.604 & 3.7 & 0.82 & 79 \\
\hline 900 & 292.6 & 14.3 & 0.879 & 1.0 & 1.07 & 90 \\
\hline 955 & 295.8 & 16.3 & 0.940 & 0.5 & 1.12 & 88 \\
\hline 1010 & 300.0 & $(18.0)$ & 1.000 & 0.0 & 1.16 & (81) \\
\hline
\end{tabular}

hydrostatic equations are applied at these layers. The continuity equation is applied at five layers of $u$, which determines $W_{l}(l=2,3$, $4,5)$ and $p_{s}$. The values of $\sigma$ at six levels where $W$ is defined are shown in Table 1 . The corresponding pressures for the basic state $\left(p_{B}\right)_{l}$ are $100,300,650,900,955$ and $1010 \mathrm{mb}$. The value of $p_{T}$ is equal to $\left(p_{B}\right)_{1}$, which is 100 $\mathrm{mb}$. The pressure for the "disturbed state" $p$ is determined from (36).

It should be remarked that the vertical arrangement of the variables may be very important to obtain valid solution of the equations. For example, solutions may not be correctly obtained if $\theta$ is defined at the same level as $\phi$, other variables $u, v$ and $W$ being defined as shown in Fig. 1. Another inappropriate model is such that $\phi$ is defined at the same level as $W$ and $\theta$ is defined at the same level as $u$ and $v$. It has been confirmed (Yamasaki, 1967; unpublished) that any solution of the finite difference equations for these schemes is not correctly obtained if the true solution corresponds to the quasi-stationary amplifying mode to which parameterized heating is essential and if the vertical grid size is not extremely small (such as $100 \mathrm{~m}$, depending on the static stability). Although this has been confirmed only for a linear solution, there is no proof that an approximate solution is obtained for a finite-amplitude problem. For this reason, the vertical grid arrangement as shown in Fig. 1 is used in this study (also used in Yamasaki (1968) apart from the difference of the vertical coordinate).

As for the horizontal grid, we use a triplynested grid system, which has been used by

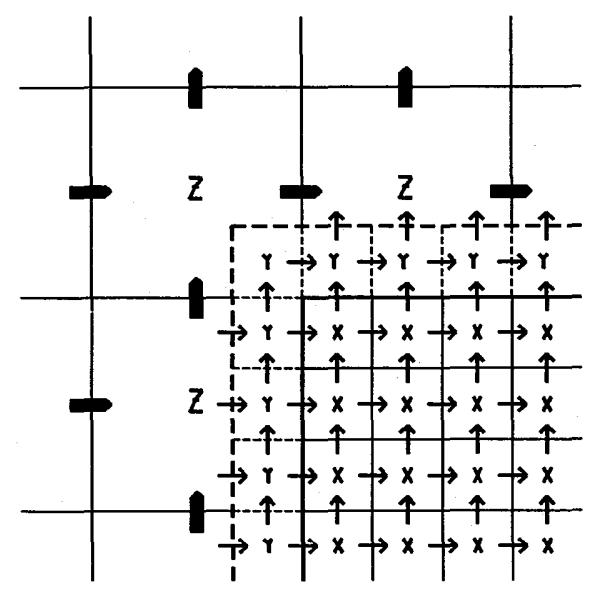

Fig. 2 Horizontal grid structure near the northwestern boundary between two grid areas. Arrows indicate locations where $u$ and $v$ are defined. At the center of each box (indicated by $\mathrm{X}, \mathrm{Y}$ and $\mathrm{Z}$ ), all variables except $u$ and $v$ are defined. The values at point $\mathrm{Y}$ and the wind velocity indicated by arrows on the dashed lines provide outer boundary conditions for the finer grid area.

many authors (e.g., Mathur, 1974 ; Madala and Piacsek, 1975; Jones, 1977 ; Ookochi, 1974, 1978). The mesh ratio is taken to be 3 . Fig. 2 shows the grid arrangement for the northwestern part of the boundary of the finer grid and the coarser grid. At the center of each box, $W, p_{s}, \phi$ and $S\left(\theta, q_{v}, q_{c}, q_{r}, Q_{r}^{c}\right)$ are defined. The arrangements of $u, v, W$ and $\phi$ are taken so that the behavior of gravity waves and unstable mode excited by parameterized heating may be properly described. At the boundary, $u$ and $v$ for the coarser grid are obtained by averaging the finer grid values of $u$ and $v$ at the boundary, respectively. The boundary conditions for $p_{s}, \phi$ and $S$ of the coarser grid are obtained by averaging the values at nine points of the finer grid. The values of $\mathrm{Y}$, which are the outer boundary conditions for the finer grid variables $\mathrm{X}$, are obtained by linear interpolation of the finer grid value $X$ and the coarser grid value $Z$. A similar interpolation is also made for $u$ and $v$. Numerical experiments have shown that even such a simple interpolation does not cause any significant noise at 
the boudary.

The domains of the fine-grid and the medium-grid are changed in accordance with the position of the tropical cyclone center, as done by many authors (e.g., Ookochi, 1978). The values at the new finer grid points are obtained from linear interpolation of the coarser grid values (and the finer grid values are also used, when necessary). The values at the new coarser grid points are obtained by averaging the finer grid point values.

As for the finite difference equations, the pressure gradient force is represented by the centered difference. The Coriolis term is obtained by averaging the values of four adjacent points. The vertical advection terms use upstream difference. The horizontal advection terms are represented by a weighted mean of the upstream difference and the centered difference. The weight of the latter is taken to be $0.6\left(C_{\mathrm{CENT}}=0.6\right)$ in this study. Since the horizontal resolution used in this study is not fine enough to describe the width of spiral rainbands properly, the use of pure centered difference $\left(C_{\mathrm{CENT}}=1\right)$ may significantly underestimate the phase velocity of the rainbands. Although the inclusion of the upstream difference tends to suppress the rainbands, quantitative discussion is not the objectives of this study. The degree of validity of the cumulus parameterization scheme used is much more important.

The time integration is performed with the combination of the leapfrog and the Euler backward scheme (Matsuno scheme). For the first 9 minutes for each period of one hour, the Euler backward scheme is used. In this case the time increment $\Delta t$ is taken to be 20 seconds not only for the fine grid but also for the medium and the coarse grids (for a grid size specified later). For the remaining 51 minutes the leap frog is used. In this case $\Delta t$ is taken to be 20 , 60 and 180 seconds for the fine, medium and coarse grids, respectively. The coarser grid value which is necessary for the outer boundary condition of the finer grid is linearly interpolated when the time level of the finer grid is different from that of the coarser grid.

\section{Initial condition and specification of model parameters}

In this study we consider a situation in which an axi-symmetric vortex exists in the atmosphere at rest. The initial tangential velocity $v_{\lambda}$ is given by

$$
\left(v_{\lambda}\right)_{l}=\left\{\begin{array}{lr}
2 v_{\max } V_{l} \frac{r}{\widehat{r}} /\left\{1+\left(\frac{r}{\widehat{r}}\right)^{3}\right\}- & C_{v}\left(\frac{r}{r_{0}}\right)^{2} \\
0 & \left(r<r_{0}\right) \\
& \left(r \geq r_{0}\right)
\end{array}\right.
$$

where $v_{\max }$ is the approximate maximum tangential velocity, $V_{l}$ represents the vertical profile of $v_{\lambda}, r$ is the radius (distance from the vortex center), $\hat{r}$ is a parameter which is related to the radius of the maximum tangential velocity, $r_{0}$ is the radius where $v_{\lambda}$ is zero and $C_{v}$ is chosen so that $v_{\lambda}$ may be zero at $r_{0}$.

The surface pressure $p_{s}$ is obtained by applying the gradient wind relation

$$
\left(f+\frac{v_{\lambda}}{r}\right) v_{\lambda}=-\frac{\partial \phi}{\partial r}-Z \frac{\partial p_{s}}{\partial r}
$$

to level 5 , assuming $\partial \dot{\phi}_{5} / \partial r=0, f=f_{0}$ and $p_{S}\left(r_{0}\right)$ $=\left(p_{S}\right)_{B}$ where $\left(p_{S}\right)_{B}$ is taken to be the surface value of $p_{B}$. The geopotential at other levels $\phi_{l}$ $(l=1,2,3,4)$ is obtained from the gradient wind relation $\left(f=f_{0}\right)$ and $\phi\left(r_{0}\right)$, which is taken to be $\phi_{B}$ (basic state geopotential). When the temperature of the basic state $T_{B}$ (and $p_{B}$ ) is given, $\phi_{B}$ is obtained from the hydrostatic equation for the basic state:

$$
\frac{\partial \phi_{B}}{\partial \sigma}=-\frac{R T_{B}}{p_{B}}\left[\left(p_{S}\right)_{B}-p_{T}\right]
$$

The temperature at the initial time is obtained from the hydrostatic equation (42). The corresponding potential temperature $\theta$ is also determined. The initial values of $u$ and $v$ are obtained from $v_{\lambda}$ by assuming that the radial velocity is zero. It is also assumed that there do not exist the vertical $\sigma$-velocity, cloud water and rainwater initially :

$$
W=q_{c}=q_{r}=q_{r}{ }^{c}=0 .
$$

The initial mixing ratio of water vapor is assumed to be uniform horizontally :

$$
q_{v}=\left(q_{v}\right)_{B}
$$

where $\left(q_{v}\right)_{B}$ is the mixing ratio of the basic state water vapor.

As shown in Fig. 1, a five-layer model ( $L=$ 
$5)$ is used, and the basic state pressure $\left(p_{B}\right)_{l}$, temperature $\left(T_{B}\right)_{l}$ and mixing ratio of water vapor $\left(q_{v}\right)_{B l}$ used in this study are shown in Table.1. For this basic state, $\sigma, z, \rho_{B}$ (basic state density) and $\left(R_{H}\right)_{B}$ (basic state relative humidity) are also shown. The numerals in the parentheses indicate the values at the uppermost and the lowest layer where $S$ is defined.

The parameters which specify the initial vortex are taken to be $v_{\max }=10 \mathrm{~m} \mathrm{~s}^{-1}, V_{l}=0.0$, $0.5,0.9,1.0$ and $1.0, \hat{r}=250 \mathrm{~km}$ and $r_{0}=1200$ $\mathrm{km}$. For these values, the radius of the maximum wind is $200 \mathrm{~km}$ and the central surface pressure is $1005.4 \mathrm{mb}$.

The parameters concerning cumulus parameterization are specified as shown in Table 2. Other physical parameters are taken to be $f_{0}=$ $5 \times 10^{-5} \mathrm{~s}^{-1}$ (corresponding to $20 \mathrm{~N}$ ), $\beta=2 \times$ $10^{-11} \mathrm{~s}^{-1} \mathrm{~m}^{-1}, C_{D}=C_{E}=0.0015, T_{\text {sea }}=301 \mathrm{~K}$, $Q_{R A D}=1 \mathrm{C} \mathrm{day}^{-1}, K_{H}=5 \times 10^{3} \mathrm{~m}^{2} \mathrm{~s}^{-1}, K_{V}=$ $10 \mathrm{~m}^{2} \mathrm{~s}^{-1}$ (layer 4 and 5), $5 \mathrm{~m}^{2} \mathrm{~s}^{-1}$ (layer 3 ) and 0 (layer 1 and 2). In addition to the vertical eddy diffusion specified above, the sensible and latent heat supplied from the sea surface (34) are assumed to be instantaneously distributed with a ratio of 4 to 6 at layers 5 and 6 ; that is $(\partial / \partial t)_{5} /(\partial / \partial t)_{6}=4 / 6$.

The horizontal grid sizes of the fine grid, $\Delta x$ and $\Delta y$, are taken to be $20 \mathrm{~km}$, and the number of grid points $N_{x}$ and $N_{y}$ is 31 . The time increment for the fine grid $\Delta t$ is 20

Table 2. Parameters concerning cumulus parameterization.

\begin{tabular}{|c|c|l|}
\hline parameter & equation & value \\
\hline$\sigma^{*}\left(z^{*}\right)$ & $(10)$ & $\sigma_{4}$ \\
$\sigma^{* *}\left(z^{* *}\right)$ & $(14)$ & $\sigma_{3}$ \\
$C_{\eta}$ & $(12)$ & $0.2(B>0)$ \\
& & $1.0(B<0)$ \\
$C^{* *}$ & $(14)$ & 0.5 \\
$E_{D}$ & $(17)$ & $5 \times 10^{-5} \mathrm{~m}^{-1}$ \\
$C_{\text {CoNv }}$ & $(20)$ & $2 \times 10^{-3} \mathrm{~m}^{-1}$ \\
$C_{R}$ & $(29)$ & $10^{-3} \mathrm{~s}^{-1}$ at $\sigma_{4}$ \\
$C_{W}$ & $(27)$ & 1 for $w>5 \mathrm{~m} \mathrm{~s}^{-1}$ \\
& & 5 for $w<0.5 \mathrm{~m} \mathrm{~s}^{-1}$ \\
$K_{D}$ & $(32)$ & $500 \mathrm{~m}^{2} \mathrm{~s}^{-1}$ for layer 4 \\
& & $170 \mathrm{~m}^{2} \mathrm{~s}^{-1}$ for layer 5 \\
\hline
\end{tabular}

seconds. As for the parameter for the advection terms we take $C_{\mathrm{CENT}}=0.6$. As also mentioned before, the time interval for use of the Euler backward scheme $t_{E B}$ and that for the leap frog $t_{L F}$ are 9 minutes and 51 minutes, respectively.

\section{Results}

Since it is assumed that an axi-symmetric vortex exists in the atmosphere at rest initially, it is not intended in this study to simulate the process of tropical cyclone formation realistically. In order to simulate this process, the large-scale environmental flow with vertical and horizontal shear should be taken into account. Furthermore, the induced asymmetry of the vortex and the behavior of convective activity may be somewhat dependent on the properties of the initially assumed vortex. The results presented in this paper are obtained for only one idealized and simplified situation. In order to get a sufficient understanding of the properties of the model tropical cyclone and to clarify the degree of validity of the parameterization scheme of cumulus convection, it is necessary that a number of numerical experiments should be performed by changing initial conditions and model parameters. This is left for the forthcoming studies. The primary objectives of the present paper is to show that the parameterization scheme is capable of simulating not only the gross features of the tropical cyclone but also mesoscale features more realistically than the schemes proposed in the past.

Fig. 3 shows the wind velocity at level 4 for the fine-grid area at 18, 30, 48 and 72 hours. The numerals on the ordinate (south-north) and the abscissa (west-east) indicate the index of the grid point (negative sign omitted). The origin of the coordinate $(0,0)$ indicates the location of the center of the initially given vortex. The fine-grid area covers a domain of $(600 \mathrm{~km})^{2}$ because the grid size is $20 \mathrm{~km}$. The areas of wind velocities larger than $25 \mathrm{~m} \mathrm{~s}^{-1}$ and $33 \mathrm{~m} \mathrm{~s}^{-1}$ are indicated by the light and the dark shade, respectively.

It is seen from Fig. 3 that the maximum velocity attains $20 \mathrm{~m} \mathrm{~s}^{-1}$ at 18 hours. The central surface pressure at this time is $1002 \mathrm{mb}$. 

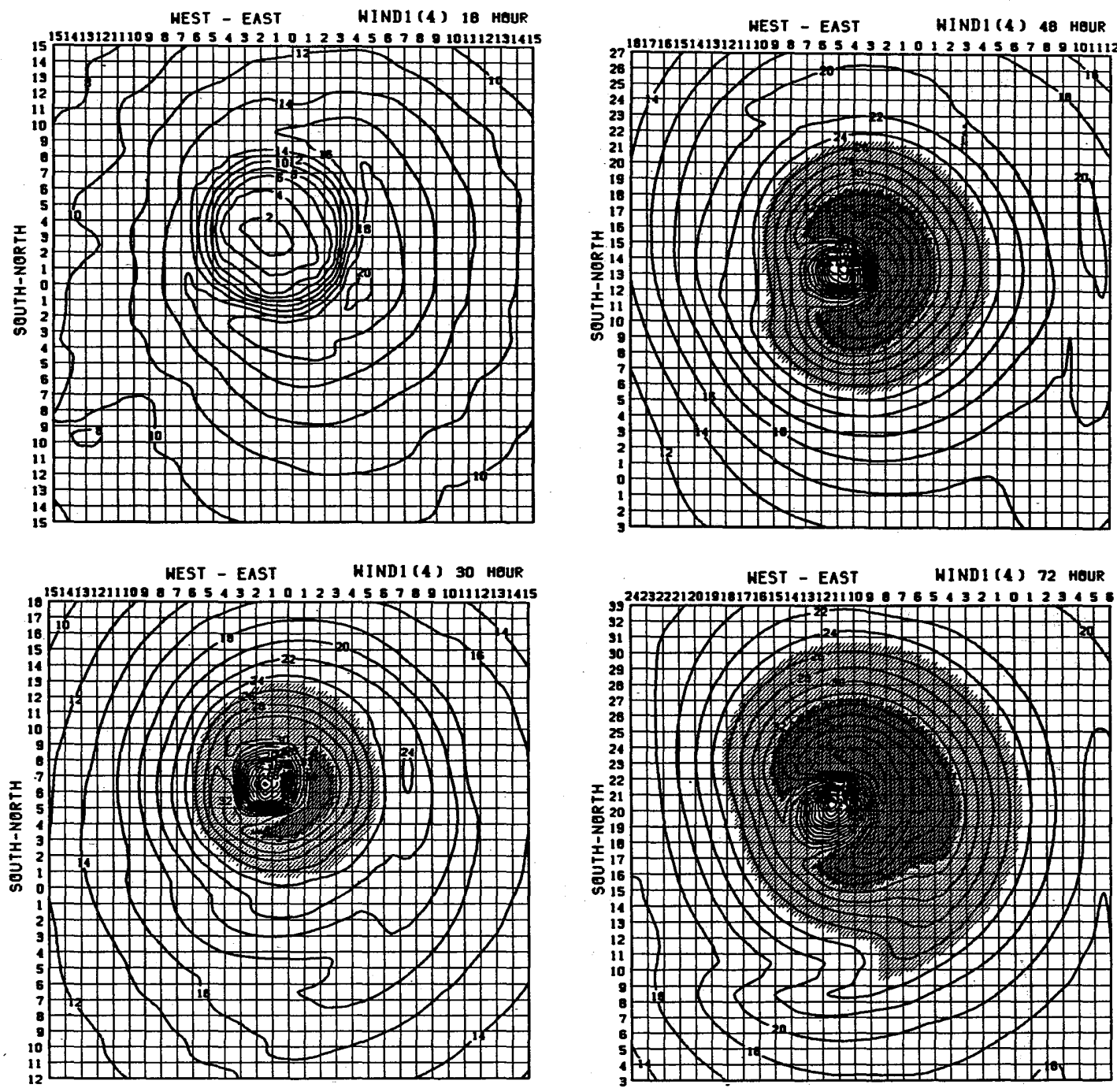

Fig. 3 Wind velocities at level 4 for the fine-grid area at 18, 30, 48 and 72 hours. Numerals on the ordinate and the abscissa indicate the index of grid points. The negative sign is omitted. The position of the initial vortex center is $(0$, 0 ). Areas of wind velocities stronger than $25 \mathrm{~m} \mathrm{~s}^{-1}$ and $33 \mathrm{~m} \mathrm{~s}^{-1}$ are shaded in two different ways.

The initially given vortex with the maximum velocity of $10 \mathrm{~m} \mathrm{~s}^{-1}$ attains the tropical cyclone intensity $\left(17 \mathrm{~m} \mathrm{~s}^{-1}\right)$ in about 16 hours. This period depends strongly on $C^{* *}$ and $C_{\eta}$. The computed tropical cyclone (hereafter referred to as TC) begins to develop rapidly after 18 hours and the hurricane intensity $\left(33 \mathrm{~m} \mathrm{~s}^{-1}\right)$ is obtained at 30 hours. The central surface pressure is $985 \mathrm{mb}$ at this time and $977 \mathrm{mb}$ at 48 hours. The maximum wind velocity of $42 \mathrm{~m} \mathrm{~s}^{-1}$ does not vary much after 48 hours, but the area of strong winds continues to expand with time until 120 hours at which the time integration is terminated. The area of winds stronger than 25 $\mathrm{m} \mathrm{s}{ }^{-1}$ extends to the $250-\mathrm{km}$ radius in the eastern sector of TC at 72 hours and $400 \mathrm{~km}$ after 108 hours. The central surface pressure is $972 \mathrm{mb}$ from 60 to 72 hours and then fall again afterwards, though very gradually. It is $968 \mathrm{mb}$ at 96 hours and $965 \mathrm{mb}$ at 120 hours. Since the 

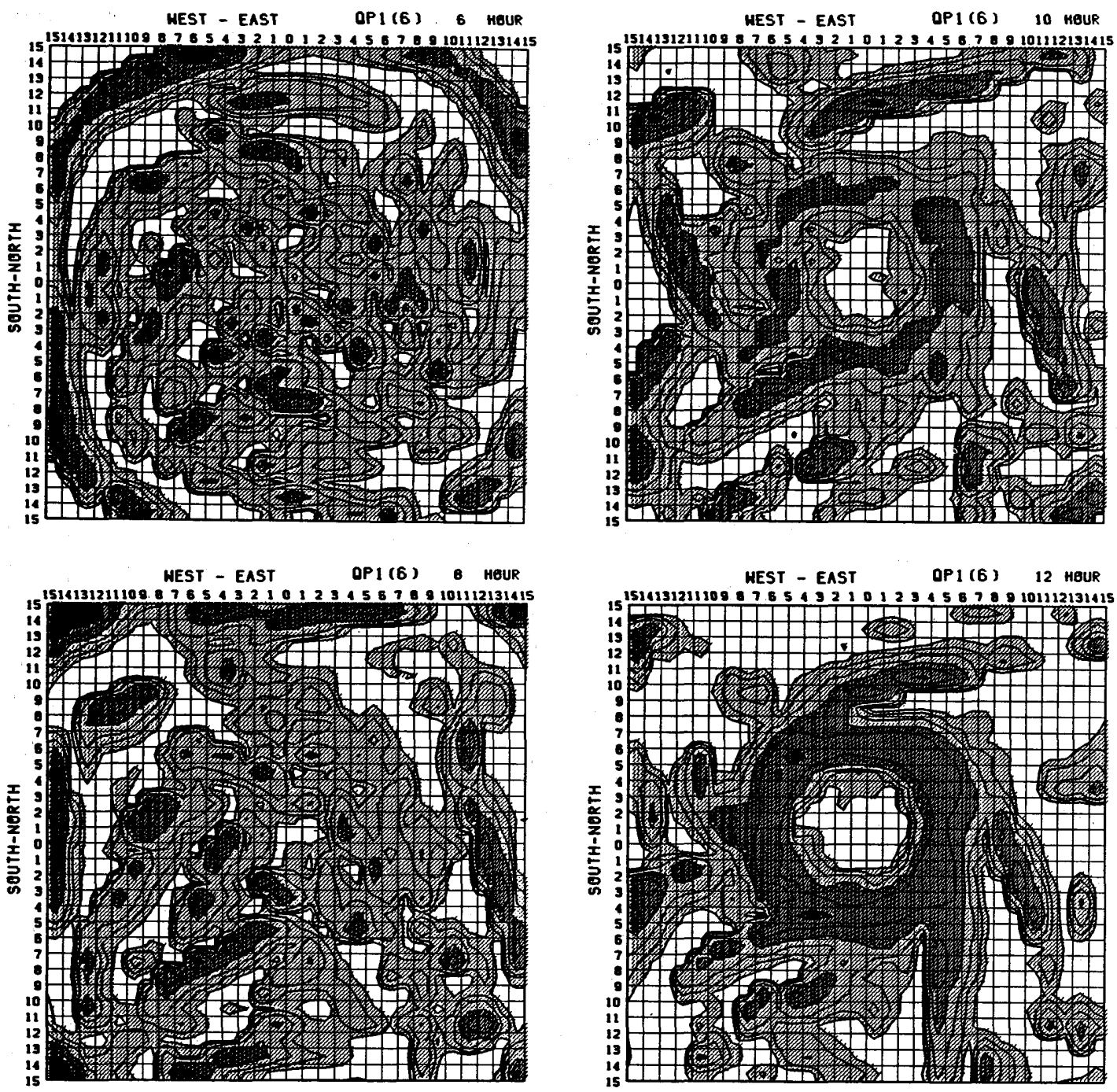

Fig. 4 Ranfall intensities $I$ at the surface for the fine-grid area at 6, 8, 10 and 12 hours. The isolines are drawn for $1,2,4,8,16$ and $32 \mathrm{~mm} \mathrm{~h}^{-1}$. The three different shades indicate $I>32,32>I>8$ and $8>I>1 \mathrm{~mm} \mathrm{~h}^{-1}$. These figures also indicate the mixing ratio of rainwater at level 6 .

grid size is $20 \mathrm{~km}$, such a large pressure fall confined near the center as obtained in $\mathrm{Y}$ cannot be described by the present model. The stages before 16 hours, from 16 to 60 hours and after 60 hours are referred to as pre-typhoon stage, development stage and mature stage, respectively:

As is also seen from Fig. 3, the location of the maximum wind tends to shift inward (toward the TC center) in the pre-typhoon and development stages. The radius of the maximum wind, which is assumed to be $200 \mathrm{~km}$ initially, is $130 \mathrm{~km}$ at 18 hours and $60 \mathrm{~km}$ at 48 and 72 hours. After TC reaches the mature stage, it shifts outwards, being $100 \mathrm{~km}$ at 120 hours. These properties are in good agreement with observations and with those obtained by the axi-symmetric models in the past. Another feature is that the location of the maximum wind rotates couterclockwise. Although such a rotation has a period of $2 \sim 3$ days in another numerical experiment (presented at the meeting 

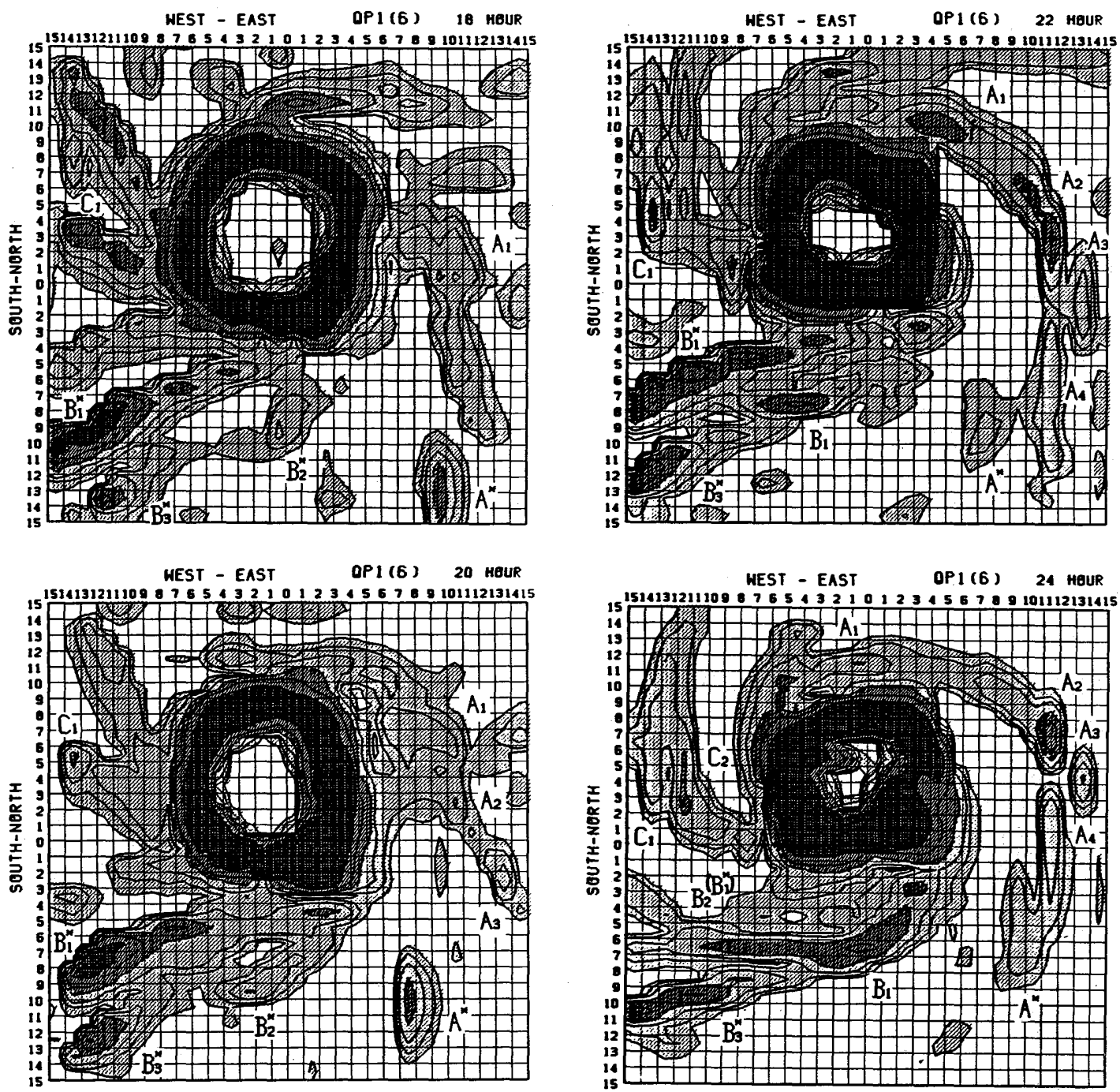

Fig. 5a Rainfall intensities at 18, 20, 22 and 24 hours. Several mesoscale convections are named.

of MSJ in 1984), the rotation in the present experiment is very slow. The feature that the maximum wind is located to the northeast of the TC center by the $\beta$-effect (Madala and Piacsek, 1975) is very pronounced in Fig. 3 . The rotation of the wind field, which is also related to the looping motion of the tropical cyclone (Yamasaki and Ohnishi, 1985), is one of the interesting problems to be studied extensively.

In the following, the behavior of mesoscale convective activities will be described. Fig. 4 shows the rainfall intensity $I$ at the surface at $6,8,10$ and 12 hours, which correspond to the pre-typhoon stage. The isolines are drawn for rainfall intensities of $1,2,4,8,16,32$ and $64 \mathrm{~mm}$ $\mathrm{h}^{-1}$. The darkest, medium and light shades indicate $I>32,32>I>8$ and $8>I>1 \mathrm{~mm} \mathrm{~h}^{-1}$, respectively. The rainfall intensity is determined from the mixing ratio of rainwater $q_{r}$ at level 6 . Therefore, Fig. 4 can also be considered as the low-level rainwater distribution. The isolines correspond to the mixing ratios of $0.064,0.12,0.22,0.41,0.76,1.40$ and $2.59 \mathrm{~g} \mathrm{~kg}^{-1}$. The area shown is the fine-grid area.

Convective activities in the model take place in the area of frictional convergence associated with the initially given vorticity field. Since the initial vortex is axi-symmetric, 

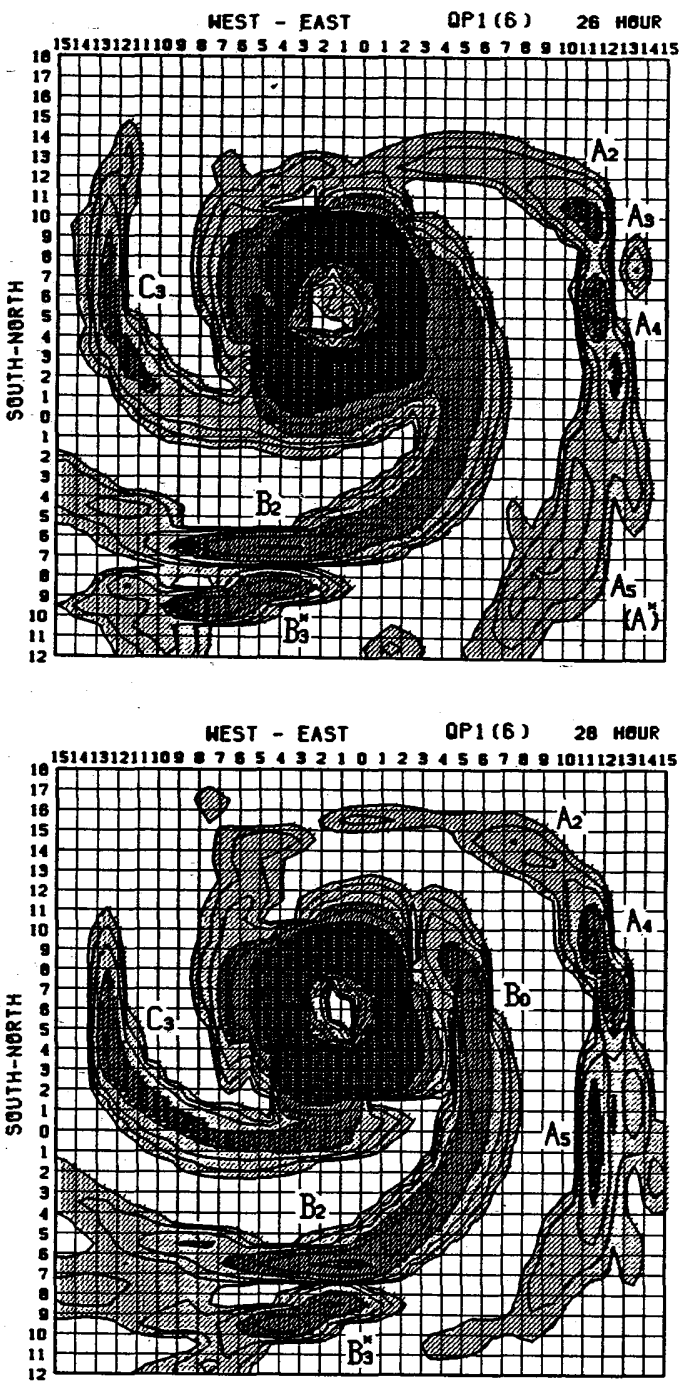
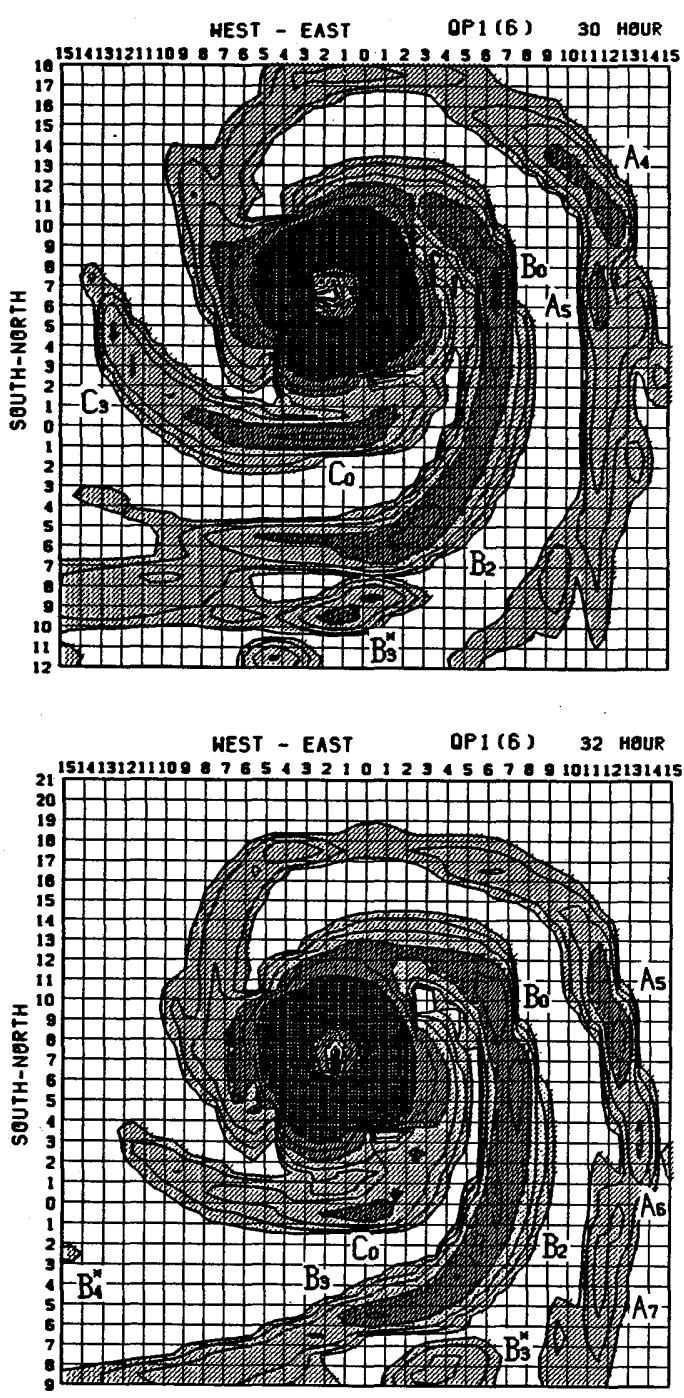

Fig. 5b Rainfall intensities at 26, 28, 30 and 32 hours.

the convergence area is nearly circular. Convective activity is most intense near the outer edge of the circular convective area. An example of this convective ring can be seen in the figure for 6 hours (although some slight asymmetry has already appeared). This convective ring propagates outward and can be clealy seen in the medium-grid area at 8 hours (not shown). These properties (and the vertical structure of the convection) are quite similar to those obtained for the non-parameterized model of $\mathrm{Y}$. In the area inside the convective ring, convective activities are quite asymmetric and take place in relatively random fashion in the very early stage. As time goes on, convective activities tend to be organized in the banded form. Some of the convections constitute an eyewall, which is still weak at this stage. The development in the pre-typhoon stage is somewhat too fast probably because relatively large values of $C^{* *}$ and $C_{\eta}$ are used, as mentioned before. The formation of an eyeeyewall system may also be slightly too early compared with the wind field probably because the initial vortex is assumed to be symmetric

* In a numerical experiment including the vertical shear of an environmental large-scale flow, it takes much time for an eyewall to form. 

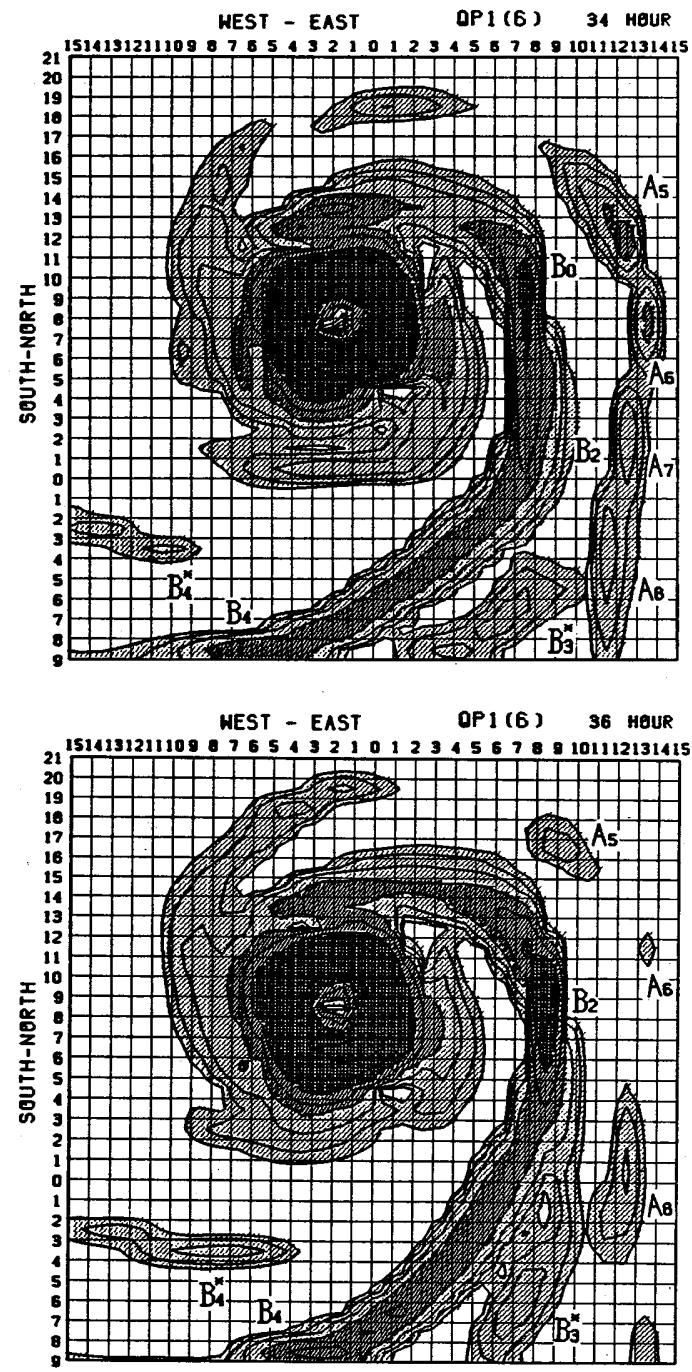

Fig. 5c Rainfall intensities at 34 and 36 hours.

and because no large-scale environmental flow is taken into account*. In spite of such shortcomings due to the situations chosen, the computed organization of mesoscale convections in the banded form is one of the important results obtained by the model. Further details will be given later.

Figs. $5 \mathrm{a}-5 \mathrm{c}$ show the rainfall intensities from 18 to 36 hours in the rapid development stage with a time interval of 2 hours. As mentioned above, these figures also indicate the patterns of the mixing ratios of rainwater at low levels. Major mesoscale convections are given such names as $A_{1}$ and $A_{2}$ in the figures.
The identification of individual mesoscale convections is based on the figures made at 1 hour intervals. The areas shown are changed at 26 and 32 hours because of the change of the fine-grid area in the time integration.

Several features can be seen from Fig. 5 . (1) The eyewall convection becomes active and the radius of the eye decreases with time, in agreement with the observed features of developing tropical cyclones. (2) The banded structure of convective activities in the area outside the eyewall is gradually changed into a spiral one. (3) Mesoscale convections form at the trailing edge of the spiral rainbands in many cases and move inward cyclonically. The movement of " $B_{3}$ " in the early stage, " $B_{1}^{* \text { " and }}$ " $A$ "” is different from this feature. (4) The lifetime of each mesoscale convection ranges from a few hours to about 10 hours (except " $B_{3}^{*}$ "). The lifetime of convections in the spiral band in the very inner area and in the eyewall cannot be defined. These properties are similar to those in the non-parameterized model of $\mathrm{Y}$. Since the fine-grid area is not large enough and the resolution is not fine enough to describe mesoscale convections and spiral rainbands properly, the identification of each mesoscale convection and quantitative discussion are not necessarily appropriate and need further studies.

Fig. 6 shows the rainfall intensities at 48,60 and 96 hours at the late development stage and the mature stage. At 48 hours, only one spiral rainband, which is a series of mesoscale convections $B_{i}$ in Fig. 5 , can be seen. The band rotates cyclonically and propagates outward, although very slowly. After 60 hours, the band is separated to the north of the TC center. These are referred to as bands (D) and (E). A new band $(F)$ is seen at 96 hours.

Fig. 7 shows the rainfall intensity for the medium-grid area at 96 hours. Three major bands can be seen. Two bands which extend to the west (D) and south (E) of the TC center are due to separation of band (B), as mentioned above. The band $(\mathrm{F})$, which forms around 70 hours, almost disappears after 110 hours. The band $(\mathrm{E})$ also begins to decay, and the northeastern portion of the band (to the east of the $\mathrm{TC}$ center) disappears after 100 hours. At this 

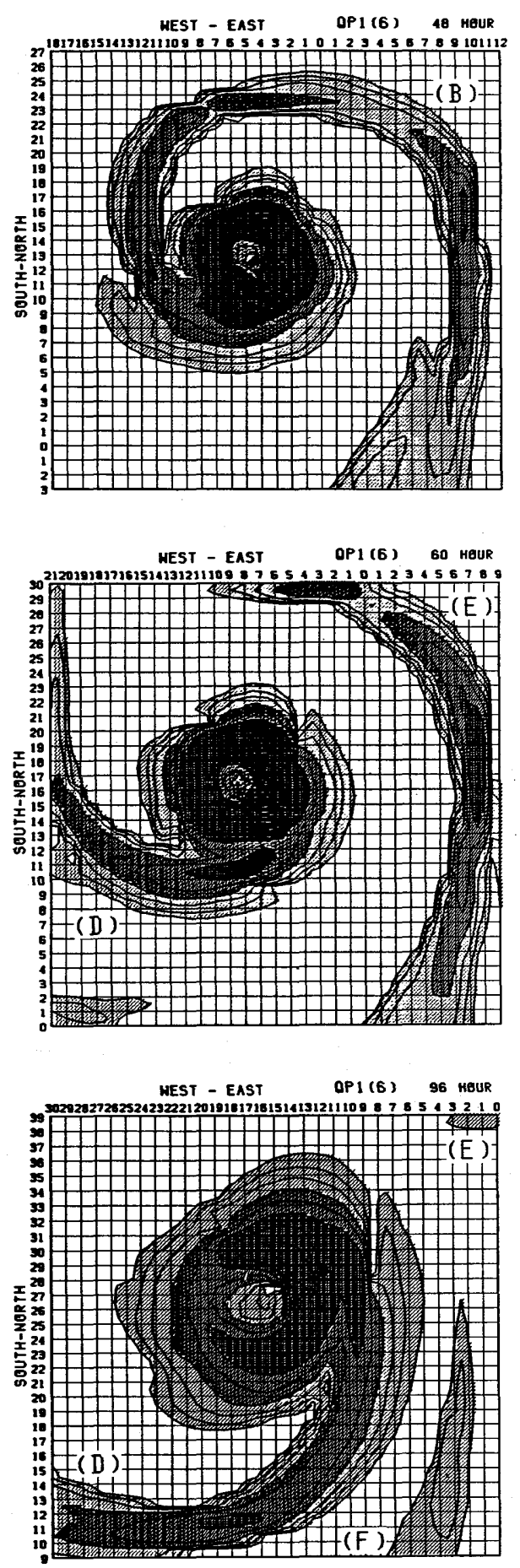

Fig. 6 Rainfall intensities at 48,60 and 96 hours. Band (B) is separated into two bands. The leading and trailing portions are referred to as (D) and (E), respectively. time the band (D) (which continues to rotate cyclonically, as other bands do) becomes the most active band. Although the grid size is very large in the medium--mesh area, it seems that rainbands are qualitatively described by the model.

Another band, which is referred to as $(G)$, can be seen to the east of the TC center. The properties of $(\mathrm{G})$ are quite different from those of the others. It propagates southward in the low-level southerly flow associated with TC. This property is similar to that of the bands in the early stage (such as " $B_{1}^{*}$ " and " $B_{3}^{*}$ " in Fig. 5). It is also similar to the tropical squall-line.

In the following, the structures of mesoscale convections and spiral rainbands will be described in more detail. It will be found that the mechanism of rainbands is essentially the same as that described in the non-parameterized model of $Y$. Figs. $8 \mathrm{a}$ and $8 \mathrm{~b}$ show the rainfall intensity (same as Fig. 4), vertical velocity at level 4 and temperature perturbations at levels 5 and 6 (levels of $S_{5}$ and $S_{6}$ in Fig. 1) at 10 hours (pre-typhoon stage).

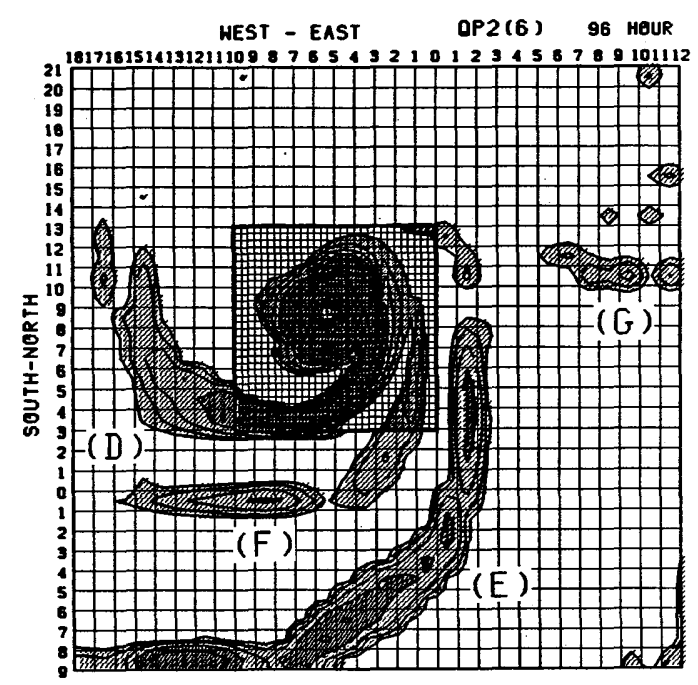

Fig. 7 Rainfall intensities at 96 hours, but for the medium-grid area. The inner square indicates the fine-grid area. Isolines are drawn for intensities of $0.5,1,2,4,8$ and 16 $\mathrm{mm} \mathrm{h}^{-1}$. Shades as well as isolines in this figure are different from those in Fig. 6. The shades are slightly different from the two grid areas because of figuring problems. 

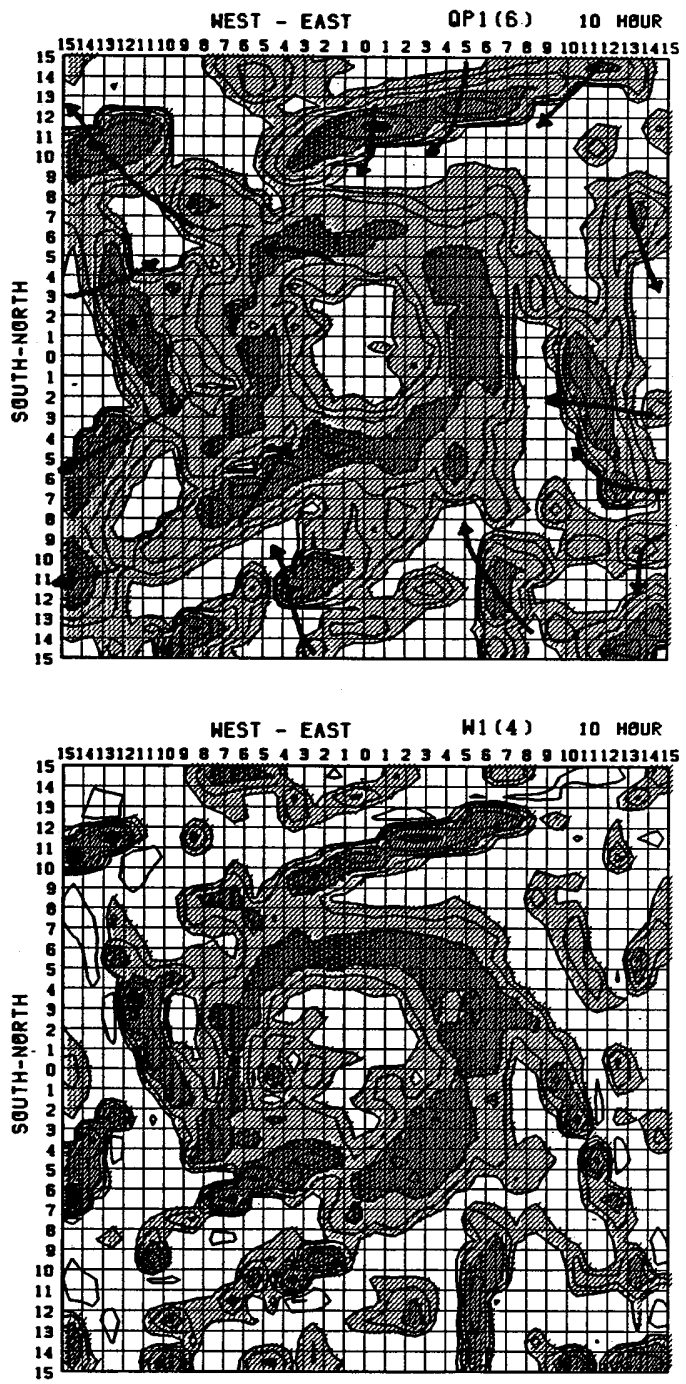

Fig. 8a Upper: Rainfall intensity for the finegrid area at 10 hours. The movements of the corresponding echo cells are indicated by the arrows. Lower: Vertical velocity at level 4 . The isolines are drawn for approximately $-5,0,2,5,10$, 20 and $50 \mathrm{~cm} \mathrm{~s}^{-1}$ (or $10^{-6} \mathrm{~s}^{-1}$ in vertical $\sigma$ velocity). Ascending areas are shaded.

Movements of individual mesoscale convections are also shown by arrows in the figure of the rainfall intensity (or low-level rainwater). These arrows are based on hourly data from 6 to 12 hours. This figure shows that there exist two types of mesoscale convections; one propagates inward (toward the TC center) and
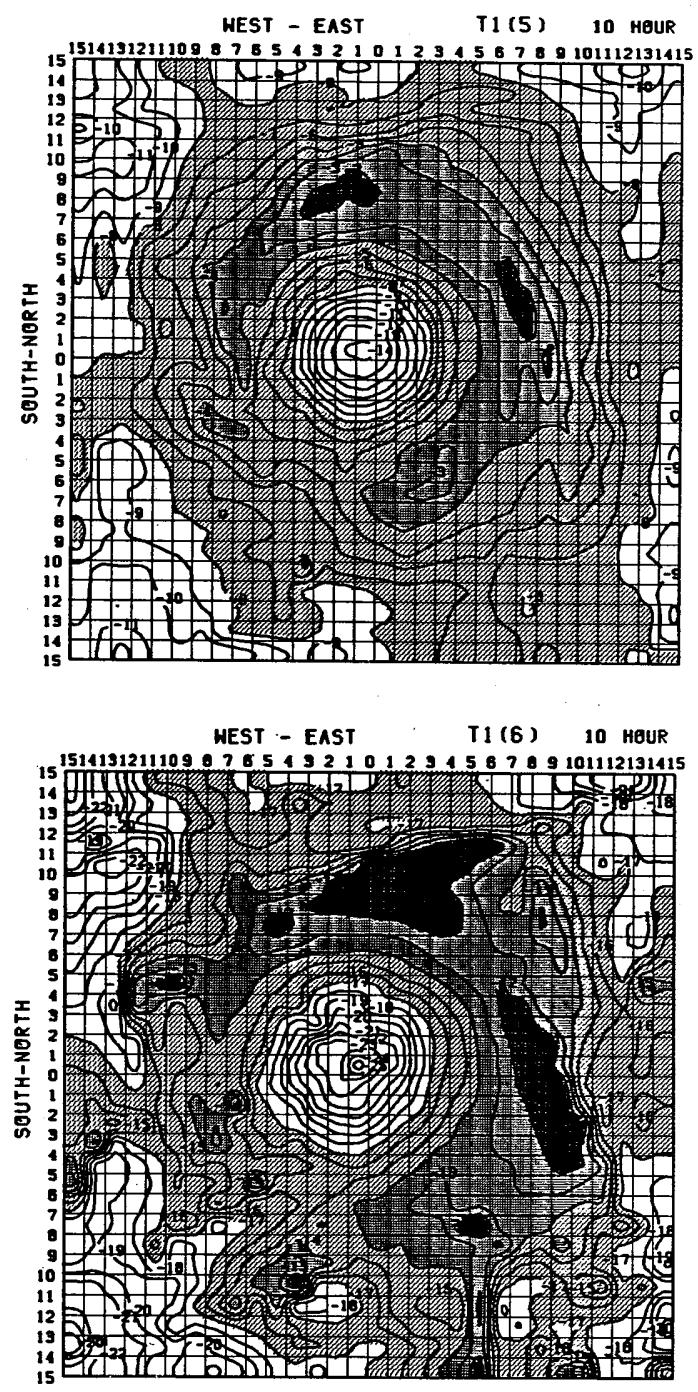

Fig. 8b Perturbation temperatures at levels 5 and 6 at 10 hours. Isolines are drawn every $0.1 \mathrm{~K}$. The unit of the numerals is also $0.1 \mathrm{~K}$. Relatively warm areas are shaded in three different ways.

the other outward. This feature was also obtained in Y. A distinct relation can be found between the propagation direction and band orientation. The bands which are directed inward and outward in the cyclonic direction propagate inward and outward, respectively. Upward motions at level 4 is located at the propagation side of the rainwater peaks. The movement of individual mesoscale convections is due to phase propagation which results from 
the formation of new convective elements in the ascending area. (The flow at this stage is still weak and therefore, horizontal advection is small in the radial direction.) The location of upward motion relative to the rainwater peak and the propagation direction are closely related to the large-scale low-level flow, which is cyclonic. As was shown in $\mathrm{Y}$, an interaction of warm moist air with cold downdraft air in the subcloud layer plays an important role in the maintenance and formation of mesoscale convections. This is clearly shown in the temperature fields in Fig. 8b. The relatively cold areas in the subcloud layer are created by evaporation of falling rainwater (except a cold area in the TC center). Upward motion and formation of new convective elements take place at the location of a large temperature gradient which is produced in the upstream side of the cold area. Thus the relations among rainwater, vertical velocity, temperature, orientation of the bands and their propagation direction are consistent with the mechanism of mesoscale convection described in $\mathrm{Y}$.

Fig. 9a shows the perturbation temperatures at levels 5 and 6 at 22 hours, which correspond to the upper right of Fig. 5a. The locations of several bands or cells are also shown by the heavy solid lines and the crosses.

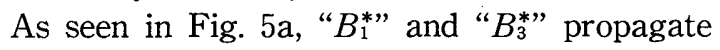
northwestward (or anticyclonically) against cyclonic flow. The temperature field associated with these convections is consistent with such a propagation. As for band (A) (consisting of mesoscale convections " $A_{1}$ ", " $\mathrm{A}_{2}$ " $\cdots$ in Fig. $5 \mathrm{a}$ ), the temperature field at 22 hours (Fig.9a) is different from the feature mentioned above. The temperature at level 5 is slightly higher in the convective area. This is because " $A_{2}$ " and " $A_{3}$ " are at the growing stage at 22 hours. Fig. $9 \mathrm{~b}$ shows the temperature fields at 30 hours, when three major spiral bands are found (upper right of Fig. 5b). It is noteworthy that the cold area is located almost at the same location as the rainband in the outer domain, while it is located at the inner edge of the rainband in the inner domain (relatively small distance from the TC center). It is important that the location of the cold area relative to the rainband changes from the outer to the inner side of the
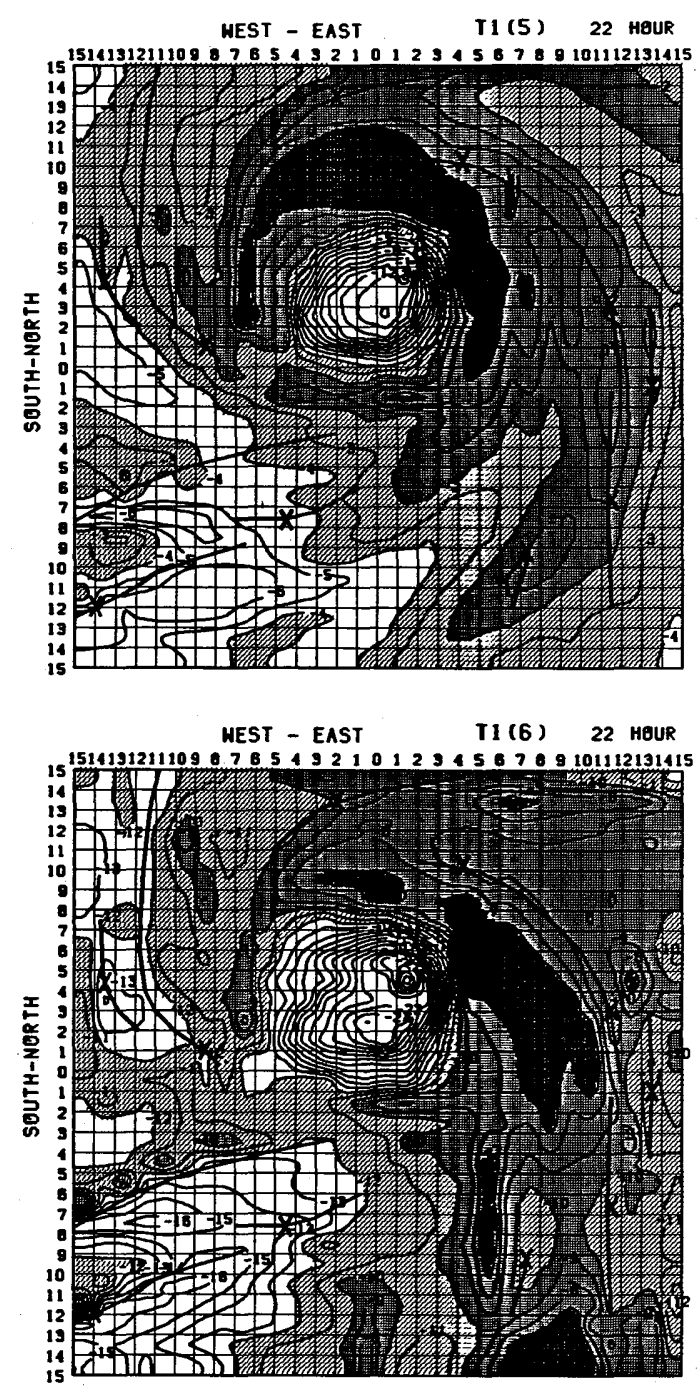

Fig. 9a Perturbation temperature (unit: $0.1 \mathrm{~K}$ ) at levels 5 and 6 at 22 hours. Relatively warm areas are shaded. Major bands are indicated by heavy solid lines, and major cells by crosses.

rainband, depending on some conditions such as frictional flow, vertical shear, rainfall intensity and conditions near the surface (relative humidity). The most important factor which causes such a change is undoubtedly frictional inflow in the present experiment. This mechanism is quite similar to that described in Y. Fig. $9 \mathrm{c}$ shows the temperature fields at 36 hours. A distinct cold area corresponding to " $B_{4}$ " in Fig. $5 \mathrm{c}$ can be seen. A weak convection " $B_{4}^{* \text { " has a }}$ 

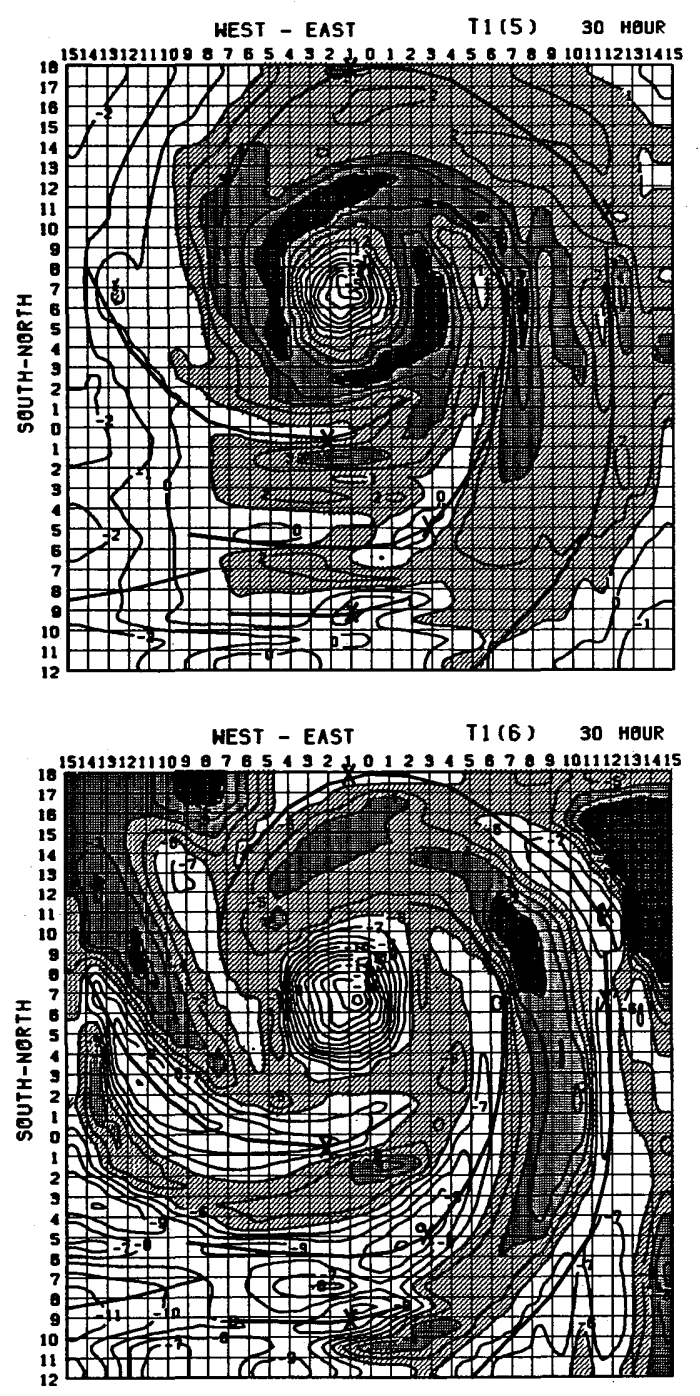

Fig. 9b As in Fig. 9a except for 30 hours.

higher temperature at level 5 and a lower temperature at level 6 . Decaying convections " $A_{5}$ " and " $A_{6}$ " have still large temperature gradients at level 6 in their outer side.

The upper part of Fig. 10a shows the vertical velocity at level 4 and the horizontal wind field at level 5 at 30 hours. The pattern of the vertical velocity field is similar to that of rainfall intensity shown in Fig. 5b. No significant difference can be found at 30 hours between the location of the maximum upward motion and maximum rainwater, although there exists a distinct difference at the early stage (Fig. 8). The ascending motion at level 5
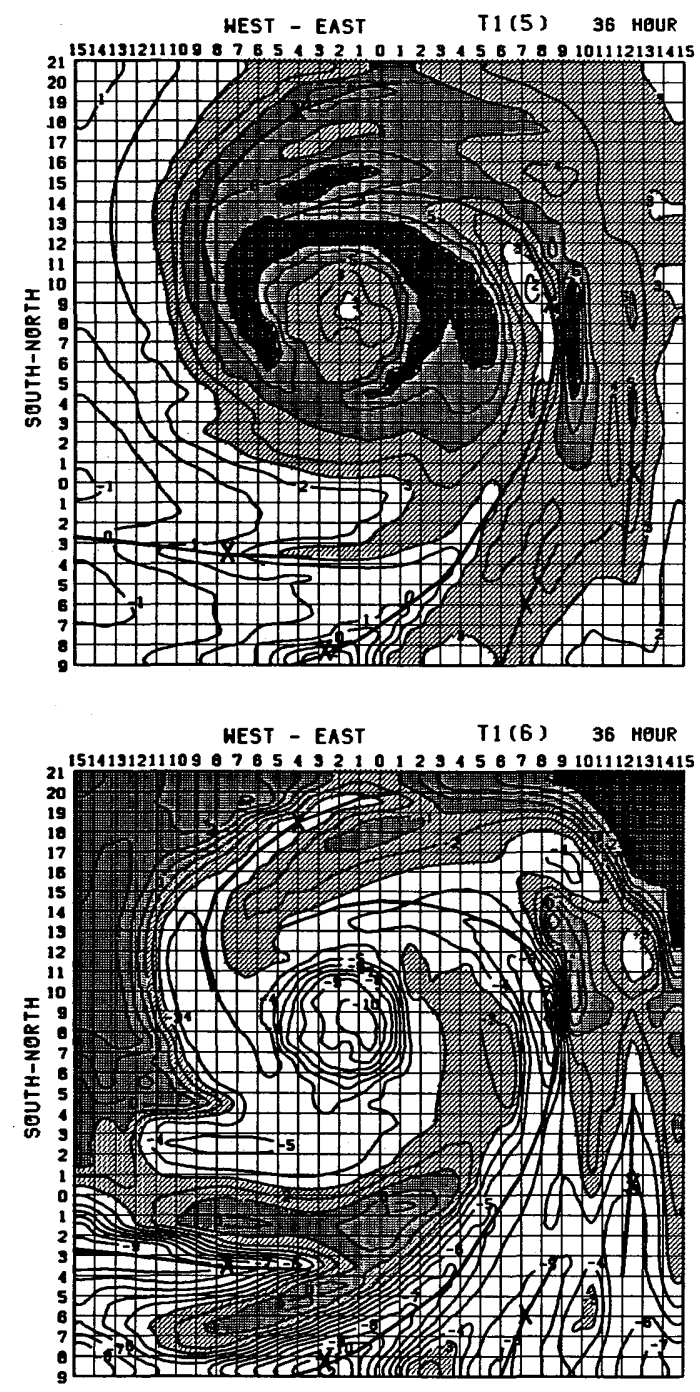

Fig. 9c As in Fig. 9a except for 36 hours.

(not shown) is broader in width and more intense at the outer side of the rainband, compared with the ascending motion at level 4 . The horizontal wind field shows that the air generally flows in the rainband from its outer side at level 5. On the contrary, the air at level 3 flows in the rainband from its inner side (not shown) because, as is well known, the flow at this level is almost in gradient wind balance and the radial component of the wind is small. The lower figure shows the wind field at level 1 . Cyclonic outflow near the TC center and anticyclonic outflow at some distance can be seen, which is typical of tropical cyclones. 

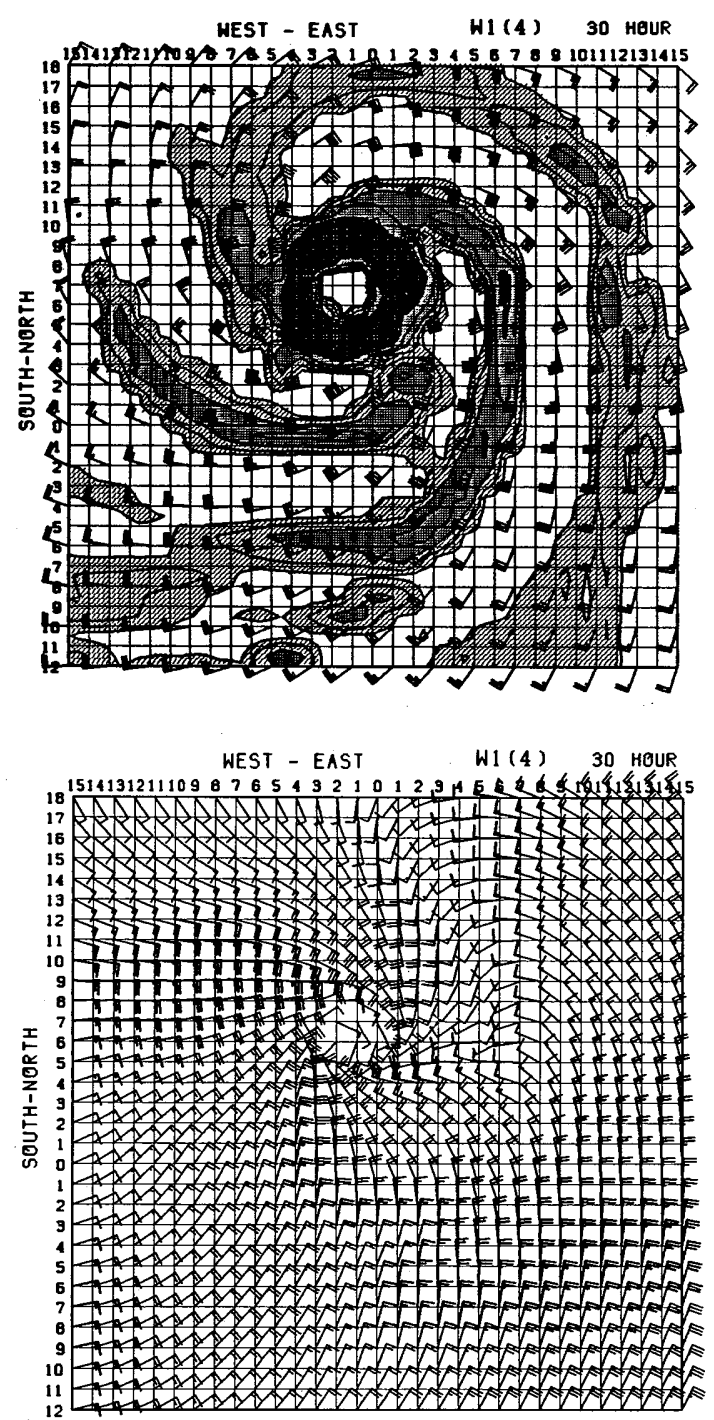

Fig. 10a Upper: Vertical velocity at level 4 at 30 hours and wind field at level 5. One full line on the wind arrow indicates the wind velocity of $5 \mathrm{~m} \mathrm{~s}^{-1}$. Lower: Wind field at level 1.

The upper part of Fig. 10b shows the temperature perturbation at level 4 . One of the important features of mesoscale convection is that the ascending area above the cloud base should be warmer than the surrounding area. The condition that $Y^{*}$ is larger than unity is necessary for the growth of mesoscale convection. However, if $Y^{*}$ is very large, the conditional instability of the "first kind" (gravitational instability) takes place. In this
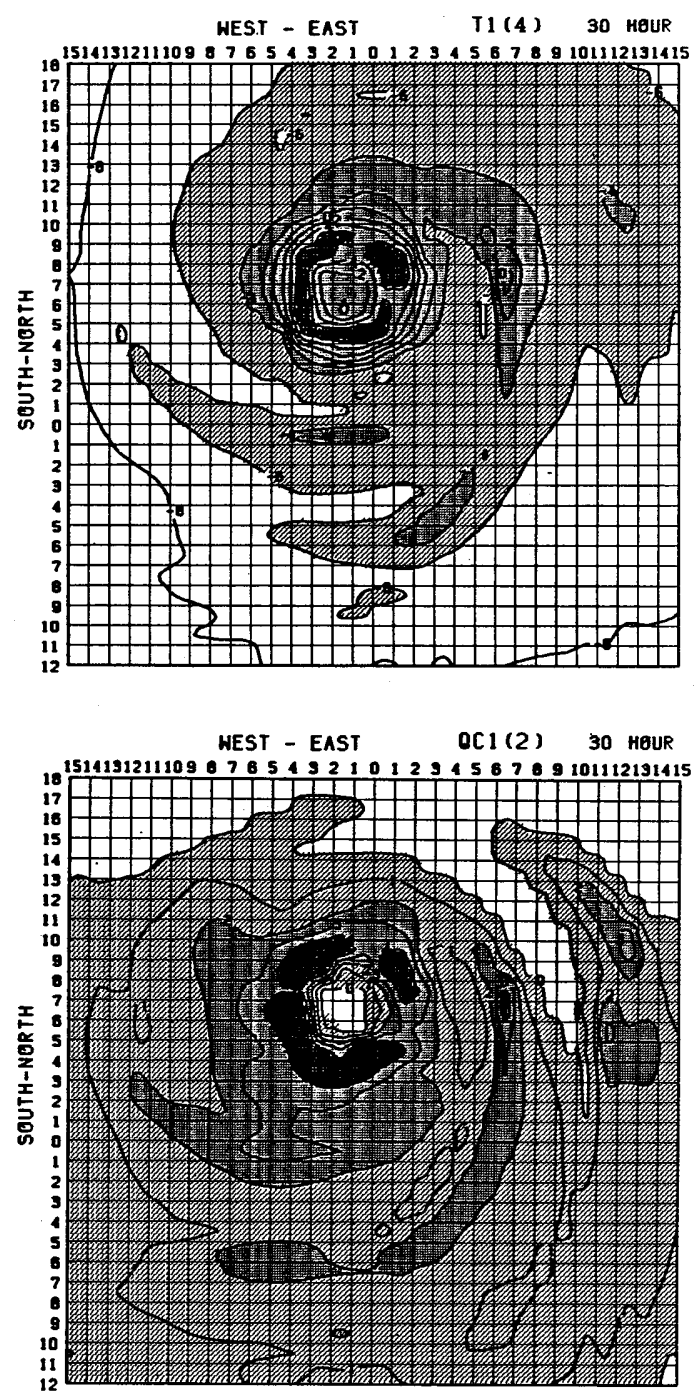

Fig. 10b Upper: Perturbation temperature (unit: $0.1 \mathrm{~K}$ ) at level 4 at 30 hours. Relatively warm areas are shaded. Lower: Mixing ratio of cloud water at level 2. Isolines are drawn every $0.1 \mathrm{~g}$ $\mathrm{kg}^{-1}$. The darkest shade indicates a mixing ratio larger than $0.4 \mathrm{~g} \mathrm{~kg}^{-1}$. Unshaded area corresponds to unsaturated area.

case convection may grow excessively or unrealistically. Although evaporation of rainwater in the subcloud layer is one of essential factors to make behaviors of explicitly resolved convection more realistic, any factor which controls the temperature rise at level 4 is also necessary. The reason why the value of $C_{R}$ in 
eq. (29) is chosen to be non-zero at $z^{*}$. (level 4$)$ is that it suppresses excessive temperature rise at level 4 and unrealistic growth of convection and that it causes eventual decay in many cases. As shown in Fig. 10b, the temperature at level 4 is relatively high in the mesoscale ascending area, but it is important that the temperature should not be very high.

The lower part of Fig. 10b shows the cloud water mixing ratio at level 2. Upward motions at level 2 (not shown) are much wider than at level 4. Not only the detrainment of cloud water from convective clouds but also the production of cloud water due to grid-scale (non-convective) condensation contribute to the pattern shown in Fig. 10. One of the noteworthy features is that a very pronounced asymmetry can be seen in the upper cloud; that is, the cloud does not extend northward very much, whereas it extends southward far away from the TC center. Such asmmetry was also seen in Fig. 7.

Fig. 11 shows the temperature fields at levels 4 and 6 at 96 hours, which corresponds to the late mature stage. The area shown is the medium-grid area. These temperature fields correspond to the rainwater field shown in Fig. 7. As mentioned above, the temperature at level 4 is higher in the growing or persisting rainbands. The upper figure confirms this property for bands (D) and (E). Band (F) is at the dissipating stage. Therefore, it is not warm in this band. As for level 6, the lowest temperature is located just at the rainband. (This holds for these three bands.) This is because frictional flow is not very intense in the medium-grid area. The southern portion of band $(\mathrm{E})$ has a large angle from the tangential direction of the TC center. The low-level wind (not shown) also has a large angle. The air flows into the band from its outer (southern) side.

As mentioned above, band $(\mathrm{G})$ behaves in a different manner. The temperature at level 6 (and 5) is higher to the south of the band because southerly flow gains much sensible heat from the sea surface. To the north of band $(G)$ convection is active and the temperature is relatively low by the effects of evaporation of rainwater. These exists a large temperature
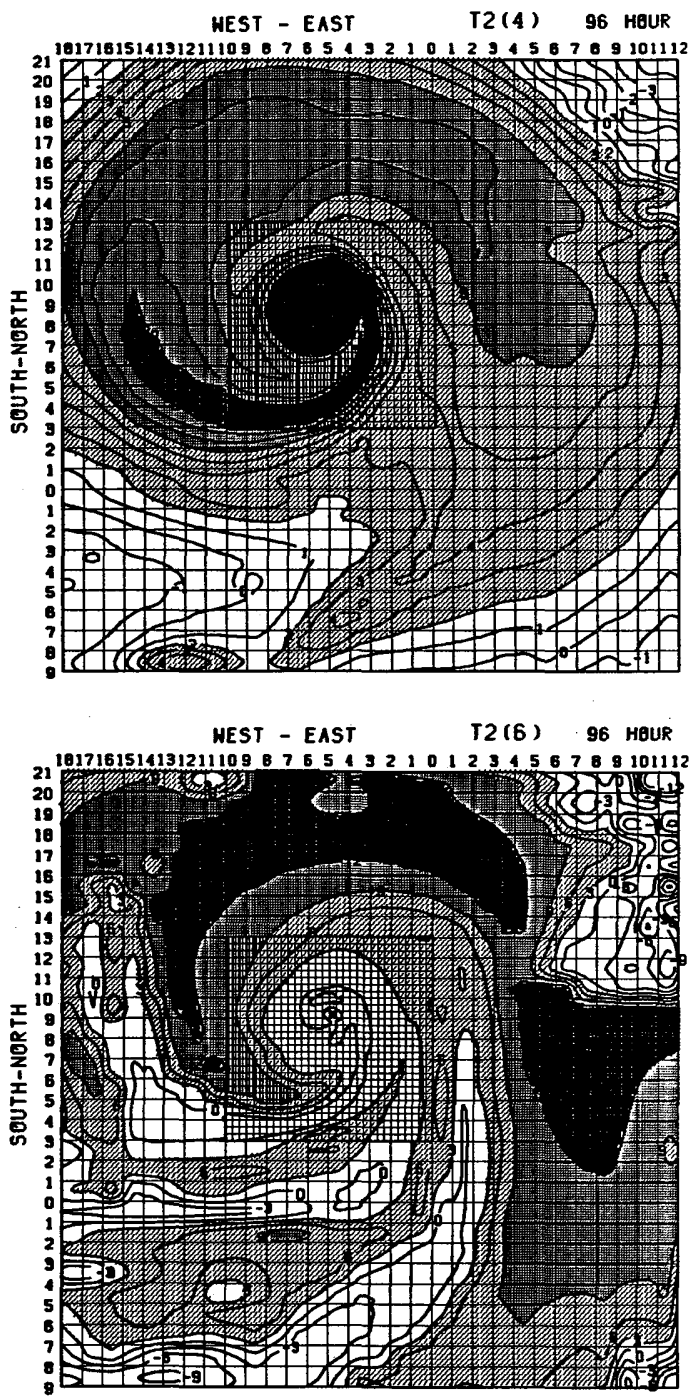

Fig. 11 Perturbation temperatures (unit : $0.1 \mathrm{~K}$ ) at levels 4 (upper) and 6 (lower) for the medium-grid area (including the fine-grid area) at 96 hours. Warm areas are shaded. The shades are slightly different for the two grid areas because of figuring problems. Isolines are drawn every $0.1 \mathrm{~K}$ and $0.3 \mathrm{~K}$ in the upper and lower figures, respectively.

gradient at the front (southern edge) of the band, which propagates southward.

In the following, some aspects of the largescale features of TC are described. Fig. 12a shows the wind fields at level 1 for the medium. grid area at 48 and 72 hours. As shown by the wind field for the fine-grid area at 30 hours (Fig. 
10a), the upper-level flow is cyclonic near the TC center and turns anticyclonic at some distance from it. The flow field at 48 hours indicates that a center of anticyclonic flow has appeared about $360 \mathrm{~km}$ west-northwest of the TC center. At 72 hours it is located about 900 $\mathrm{km}$ west-southwest of the TC center (at the western edge of the medium-grid area). Although the upper-level flow is cyclonic near the TC center, the flow field for the fine-grid area (not shown) indicates that the cyclonic flow is almost confined within a $100-\mathrm{km}$ radius.
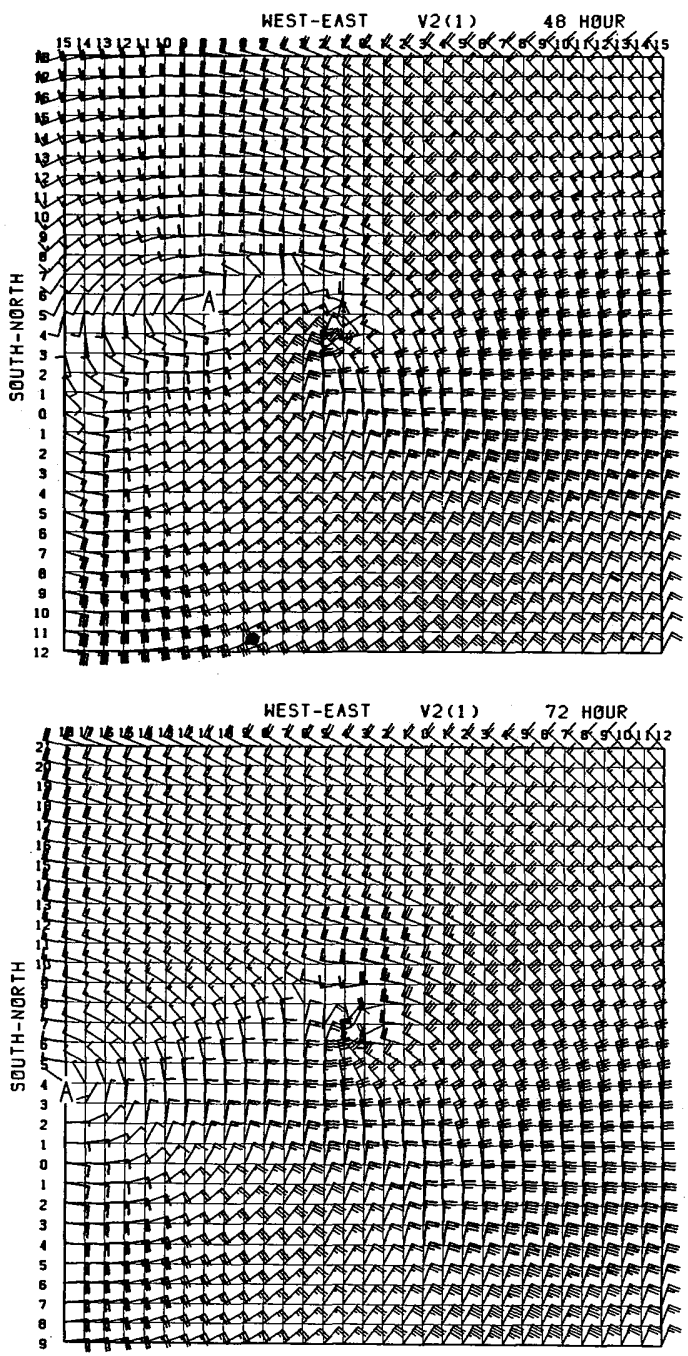

Fig. 12a Wind fields at level 1 for the mediumgrid area at 48 and 72 hours. A center of anticyclonic flow is indicated by $A$.
Roughly speaking, the upper-level flow over the TC center is northerly, as indicated by the figure for the medium-grid area. Anticyclonic flow is most intense to the southeast of the TC center and its maximum velocity attains $22 \mathrm{~m}$ $\mathrm{s}^{-1}$. This is shown in Fig. 12b, which indicates the wind velocity. The minimum of wind velocity located at $(-3.5,6.5)$ corresponds to the TC center. This is also the center of the cyclonic flow, which can be clearly seen only for the wind field for the fine-grid area (not shown). Another minimum, located at the western edge of the domain, corresponds to the center of the anticyclonic flow.

Fig. 12c shows the perturbation temperatures at levels 2 and 3 and the relative humidity at level 2 at 72 hours. The temperature fields are shown for the fine-grid area and the relative humidity for the medium-grid area. It is seen that the so-called upper tropospheric warm core is well simulated. A maximum temperature anomaly is about $13 \mathrm{~K}$. Although the warm area is much more circular at upper levels than at lower levels, it extends rather westward (northwestward at 48 hours). This feature is consistent with the location of the anticyclonic flow center shown in Fig. 12a. It is also seen from Fig. 12c that the relative

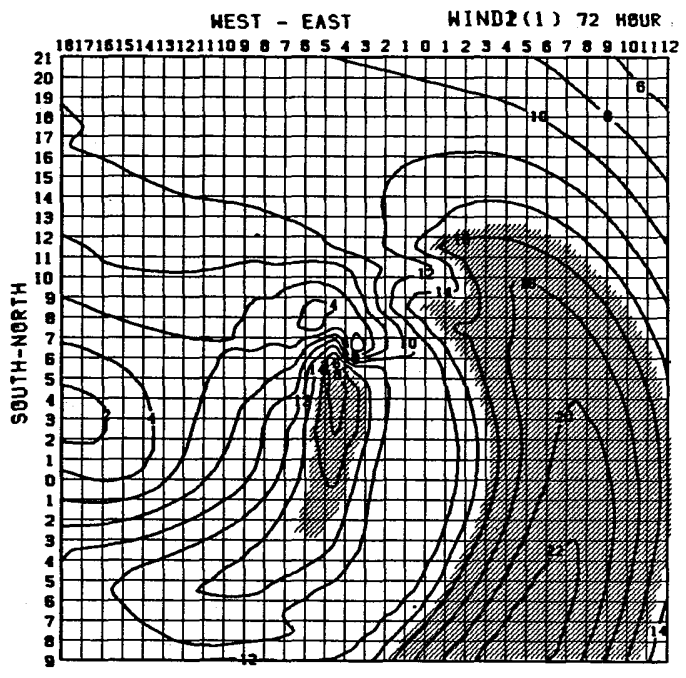

Fig. 12b Wind velocity at level 1 , corresponding to the lower part of Fig. 12a. Areas of wind velocity stronger than $15 \mathrm{~m} \mathrm{~s}^{-1}$ are shaded. 

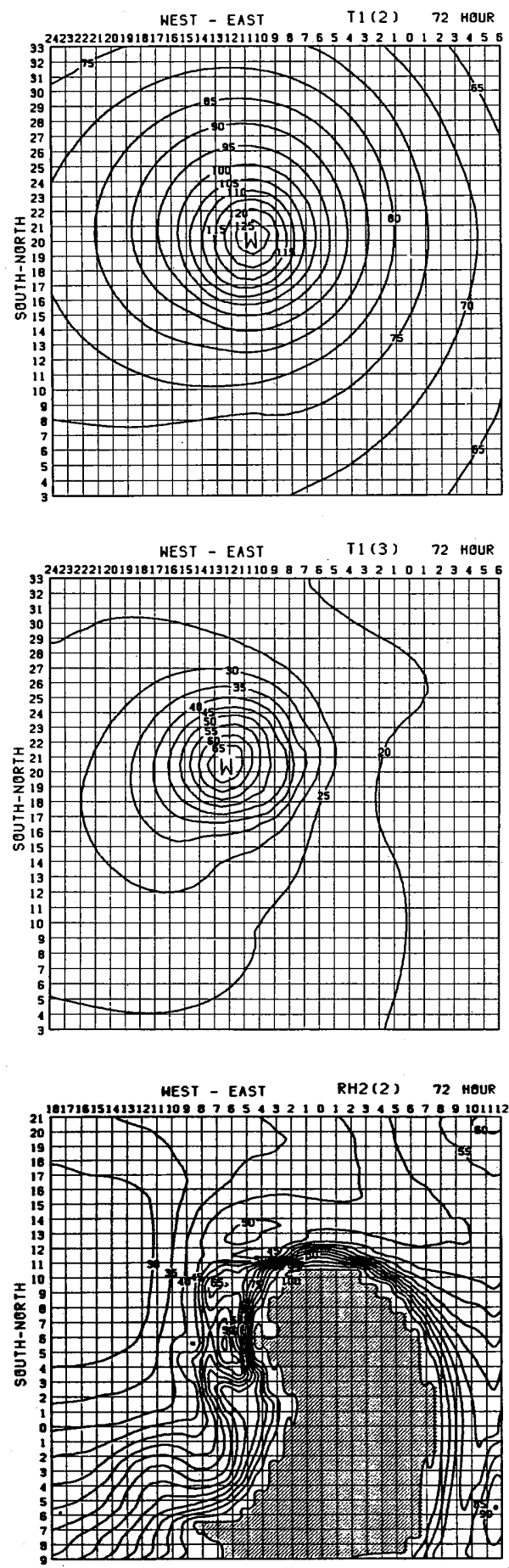

Fig. 12c Perturbation temperatures (unit: $0.1 \mathrm{~K}$ ) at levels 2 and 3 for the fine-grid area and relative humidity at level 2 for the medium-grid area. Saturated area is shaded. Its unsmoothed boundary line is due to figuring problems. humidity at level 2 is very low to the northwest of the TC center, which is located at $(-3.5,6.5)$, that is, near the northwestern boundary of the upper-level cloud shield. There exists a sharp gradient of the relative humidity near the boundary of the upper-level cloud, particularly at the northern and northwestern boundaries. The cloud extends eastward and southward far away from the TC center. These features may be typical of a tropical cyclone which moves north-northwestward owing to the latitudinal variation of the Coriolis parameter ( $\beta$-effect).

\section{Discussion and concluding remarks}

A three-dimensional tropical cyclone model has been developed in this study. The most important aspect of the model lies in an attempt to simulate mesoscale features in the tropical cyclone by treating cumulus convection implicitly. In this respect, the model seems to be similar to many models proposed in the past. However, mesoscale features obtained in this study are very different, particularly those of spiral rainbands as well as other bands at the early development stage. As mentioned in section 1, most modeling studies (e.g., Anthes, 1972 ; Kurihara and Tuleya, 1974) have interpreted the spiral rainbands as a kind of internal gravity wave excited by cumulus convection, and in fact, rainbands in their models behave like a gravity wave, as seen in the vertical structure and phase velocity. As shown in section 6 , the rainbands obtained from the present model do not behave like a gravity wave. The structure and the mechanism of the computed rainbands are similar to those obtained by the non-parameterized model of Y.

Observations from the satellite and the radar have indicated that most spiral rainbands do not propagate so fast as gravity waves do. The horizontal structure of the rainbands analyzed by Tatehira $(1961,1962)$ is much more similar to that of the rainbands obtained in this study than in other modeling studies (, although only the structure near the surface was available in his observational studies). Similarities can be found in the temperature, pressure and wind fields in and around the rainband. The temperature near the surface is relatively high in the outer side of the rainband and low in the 
inner side. The warm air near the surface flows into the rainband from its outer edge. This feature has also been observed by Barnes et al. (1983). The horizontal variation of the equivalent potential temperature in the subcloud layer is qualitatively similar to that of Barnes et al. (1983). Quantitatively, however, the results from the non-parameterized model of $\mathrm{Y}$ are much more similar to Barnes et al. This also holds for the wind field. This may be improved by changing the values of the model parameters such as $C^{* *}$ and $C_{\eta}$, which control the intensity of mesoscale convection. The location of the formation of mesoscale convections and their movement are also similar to those of echo cells described by Tatehira(1961), although echo cells corresponding to cumulus convection are not treated explicitly in this study. A trend of the gradual transition from convective to stratiform precipitation toward the downwind portion of the band, as described by Barnes et al., can be found in the present model. Unfortunately, the entire structure of the rainbands cannot be compared with observations because of a lack of appropriate observations.

In the case of rainbands in which frictional inflow is not very strong, warm moist air flows in the bands from the inner edge. In this case the band orientation has a large angle with the tangential direction. The most extreme case is the transverse band $\left(B_{1}{ }^{*}\right.$ in Fig. $5 \mathrm{a}$ and $\mathrm{G}$ in Fig. 7). An observational example of the rainbands in which warm air flows from the inner edge has been given by Ishihara et al. (1986).

The present model also simulates an eye and eyewall. The size of the eye decreases in the development stage and increases in the mature stage, which has usually been observed in real tropical cyclones and also simulated by many models in the past. Although the change of the vertical tilt of the eyewall and relative locations among a maximum upward velocity in the eyewall, a maximum tangential velocity and rainwater are also qualitatively simulated, the features obtained from $\mathrm{Y}$ are much more similar to observed eyewall features (e.g., Jorgensen, 1984). More realistic simulation (including asymmetric structure) may be made with finer horizontal resolution of the numerical model.

As for the mesoscale features at the formative stage, a comparison of the model results with observations is almost impossible. This is because radar observations have not clarified mesoscale features at this stage and because the present model does not include the vertical shear of the large-scale environmental flow, which usually exists in the real situation. Convective activities may be greatly modified by this effect (Yamasaki, 1984). A preliminary result will be reported in a forthcoming paper.

Some of the properties of TC simulated in this study are those which are not much affected by the cumulus parameterization scheme used. As shown by Madala and Piacsek(1975), the movement of TC toward NNW (or NW) and the maximum wind velocity located to the east or northeast of the TC center are due to the $\beta$-effect. The position of the TC center at 96 hours is $(-16,26)$, as seen from Fig. 6. The average speed of the movement is $150 \mathrm{~km} \mathrm{day}^{-1}$ (about $2 \mathrm{~m} \mathrm{~s}^{-1}$ ). Another feature of TC is that an upper tropospheric anticyclonic circulation center (Fig. 12a) and also a high pressure center (not shown) are found to the northwest or the west of the TC center. This feature cannot necessarily be inferred from the axi-symmetric model in which an upper high pressure area takes the form of a ring at some distance from the TC center. Corresponding to the upper pressure field, the upper tropospheric warm area extends northwestward or westward, and the upper layer is very dry in the same portion of TC (Fig. 12c). Such features of temperature, pressure and water vapor fields were pointed out by Kitade(1980). It is suggested that the warming and drying in the western and northwestern portion of TC are due to large-scale downward motion in the upper high pressure area. A very strong anticyclonic outflow in the southeastern portion and a strong outflow just to the south of the TC center (Figs. 12a and 12b) seem to be also typical of tropical cyclones subject to the $\beta$-effect under no (or weak) vertical shear of an environmental flow.

In this paper the results from only one 
numerical experiment are described. In order to improve its original version of the model (presented at the meeting of MSJ in 1981) and to understand dependencies of the properties of computed tropical cyclones on the model parameters and the ways of modeling, a number of numerical experiments have been performed. One of the most difficult problems has been how $Y^{*}$ should be determined. Some assumptions different from (14) may be possible. For example, it might be reasonable to assume that $Y^{*}$ should be related to the relative humidity at $z^{*}$. However, attempts along this line have not been successful. An attempt to predict the temperature change at $z^{*}$ from the large-scale vertical motion (including grid-scale condensation process) has not worked well. The assumption as to $\eta$ in the case of negative $B$ in (12) or the choice of $C_{\eta}$ as shown in Table 2 are also important to avoid unrealitic behavior at the later development stage and the mature stage. If $\eta$ is assumed to be unity even when $B$ becomes negative, an excessive temperature rise occurs. This property cannot be inferred from the linear theories (Ooyama, 1964 ; Syono and Yamasaki, 1966). This is closely related to the vertical profile of the vertical motion (Yamasaki, 1983), which is rather dynamically determined in the eyewall area. If $\eta$ is taken to be zero for negative $B$, oscillatory motions with large amplitudes take place. The present choice of $C_{\eta}$ assures the continuous change of $\eta$, which is necessary to avoid such oscillatory motions. The assumption for the effects of convective downdraft is also an important point. The assumption (32) is only a crude approximation even in the qualitative sense. The vertical velocity of the air and the fall velocity of rainwater relative to the air in the clouds are important factors in determining the properties of mesoscale convection, particularly its time scale. In the present model the parameterized fall of rainwater appears to be somewhat too fast because mesoscale convection (not cumulus convection) is to be simulated. Neglect of the ice (or solid) phase is also responsible for the properties of mesoscale convection. Another factor is the vertical transport of momentum. Although this effect is partly included in the form of the grid-resolved vertical transport, more realistic incorporation will improve the behavior of mesoscale convection.

One of the unsatisfactory aspects of the present parameterization scheme seems to be that the form of eqs. (1) (4) is used. This is based on the equations derived by Ooyama (1971) and Arakawa and Schubert(1974), assuming that the cloud fraction is small. Since this assumption is not appropriate when mesoscale convection is to be simulated, this aspect of the present model should be improved. Nevertheless, the model appears to describe the essential mechanisms of tropical cyclones simulated in $\mathrm{Y}$. This is probably because the above assumption is the only "quantitative" shortcoming at the early development stage and because the finally assumed form (in other words, the combined form of (1) (4) with (10) $\sim(14)$ ) in the case of $S_{c} \rightarrow S$ (nearly moist neutral stratification) is reasonable to some extent at the later development and mature stages. In the latter case, it can be interpreted in such a way that the assumption of small cloud fraction is not used. Another important point to be improved, which is related to the above, is the necessity of predicting the cloud fraction. This is also important for quantitative estimation of rainwater which falls in the ascending air in the cloud because the vertical velocity can be estimated in a more reasonable way. The present determination of water vapor and cloud water in the parameterized convective clouds $q_{v}^{c}$ and $q_{c}^{c}$ should also be improved. These shortcomings of the model and the necessity of their improvements were noticed at an early stage of this study. An attempt to improve these aspects is being made.

In conclusion, the present model contains many uncertain assumptions and parameters and also shortcomings. These should be improved by further numerical experiments and theoretical considerations and by further understanding of the processes which really occur in observed tropical cyclones.

Acknowledgements : This work has been made as part of a five-year plan of the development of a three-dimensional tropical cyclone model in MRI, which started in 1980. The 
author wishes to thank Dr. A. Katayama, Dr. M. Aihara, Mr. K. Masamura and the staff members of the Typhoon Research Division for their support of this study. The author also wishes to thank Prof. T. Matsuno for encouragement and valuable discussions. Numerical calculations have been made with the use of HITAC M200H and S810 of the Meteorological Research Institute.

\section{References}

Anthes, R.A., 1972 : Development of asymmetries in a three-dimensional numerical model of the tropical cyclone. Mon. Wea. Rev., 100, 461-476.

- , S.L. Rosenthal, and J.W. Trout, 1971 : Preliminary results from an asymmetric model of the tropical cyclone. Mon. Wea. Rev., 99, 744-758.

Arakawa A. and W.H. Schubert, 1974 : Interaction of a cumulus cloud ensemble with the large-scale environment. Part I. J. Atmos. Sci., 31, 674-701.

Barnes, G.M., E.J. Zipser, D. Jorgensen and F. Marks, JR., 1983: Mesoscale and convective structure of a hurricane rainband. $J$. Atmos. Sci., 40, 2125-2137.

Byers, H.R. and R.R. Braham, 1949: The Thunderstorm. U.S. Dept. of Commerce, $287 \mathrm{pp}$.

Charney, J.G. and A. Eliassen, 1964 : On the growth of the hurricane depression. $J$. Atmos. Sci., 21, 68-75.

Ishihara, M., Z. Yanagisawa, H. Sakakibara, K. Matsuura and J. Aoyagi, 1986: Structure of a typhoon rainband observed by two doppler radars. (to be published in $J$. Meteor. Soc. Japan.)

Jones, R.W., 1977 : A nested grid for a threedimensional model of a tropical cyclone. $J$. Atmos. Sci., 34, 1528-1553.

Jorgensen, D.P., 1984 : Mesoscale and convective-scale characteristics of mature hurricanes. Part I : general observations by research aircraft. J. Atmos. Sci., 41, 1268-1285.

Kessler, E., 1969: On the distribution and continuity of water substance in atmospheric circulation. Meteor. Monographs,
10, No. 32, Amer. Meteor. Soc., 84 pp.

Kitade, T., 1980: Numerical experiments of tropical cyclones on a plane with variable Coriolis parameter. J. Meteor. Soc. Japan, $58,471-488$.

Kuo, H.L., 1965 : On the formation and intensification of tropical cyclones through latent heat release by cumulus convection. $J$. Atmos. Sci., 22, 40-63.

Kurihara, Y., 1975: Budget analysis of a tropical cyclone simulated in an axisymmetric numerical model. J. Atmos. Sci., 32, 25-59.

- , and R.E. Tuleya, 1974: Structure of a tropical cyclone developed in a threedimensional numerical simulation model. J. Atmos. Sci., 31, 893-919.

- , and M.A. Bender, 1982: Structure and analysis of the eye of a numerically simulated tropical cyclone. J. Meteor. Soc. Japan, 60, 381-395.

Madalą, R.V. and A.A. Piacsek, 1975 : Numerical simulation of asymmetric hurricane on a $\beta$-plane with vertical shear. Tellus, 27, 453-468.

Mathur, M.B., 1974 : A multiple-grid primitive equation model to simulate the development of an asymmetric hurricane (Isbell, 1964). J. Atmos. Sci., 31, 371-393.

_ 1975 : Development of banded structure in a numerically simulated hurricane. $J$. Atmos. Sci., 32, 512-522.

Miller, B.I., P.P. Chase and B.R. Jarvinen, 1972 : Numerical prediction of tropical weather systems. Mon. Wea. Rev., 100, 825-835.

Ogura, Y., 1964: Frictionally controlled, thermally driven circulations in a circular vortex with application to tropical cyclones. J. Atmos. Sci., 21, 610-621.

Ookochi, Y., 1974: Numerical prediction of typhoon movement with use of multiple grid nesting. J. Meteor. Soc. Japan, 52, 387390 .

_ 1978 : Preliminary test of typhoon Irma using a moving multiple-nested grid (MNG). J. Meteor. Soc. Japan, 56, 571-582.

Ooyama, K., 1964 : A dynamical model for the study of tropical cyclone development. Geofisica Internacional (Mexico), 4, 187-198. — 1969 : Numerical simulation of the life 
cycle of tropical cyclones. J. Atmos. Sci., $26,3-40$.

-, 1971: A theory on parameterization of cumulus convection. J. Meteor. Soc. Japan, 49 (special issue), 744-756.

,- 1982 : Conceptual evolution of the theory and modeling of the tropical cyclone. $J$. Meteor. Soc. Japan, 60, 369-380.

Rosenthal, S.L., 1970 : A circularly symmetric primitive equation model of tropical cyclone development containing an explicit water vapor cycle. Mon. Wea. Rev., 98, 643-663.

Syono S. and M. Yamasaki, 1966 : Stability of symmetrical motions driven by latent heat released by cumulus convection under the existence of surface friction. J. Meteor. Soc. Japan, 44, 353-375.

Takeda, T., 1971: Numerical simulation of a precipitating convective cloud: the formation of a "long-lasting" cloud. J. Atmos. Sci., 28, 350-376.

Tatehira, R., 1961: A mesosynoptic and radar analysis of typhoon rain band, case study of typhoon "Helen", 1958. Proc. Second Tech. Conf. Hurricanes, Amer. Meteor. Soc., 115126.

__, 1962: Radar and meso-scale analysis of rainband in Typhoon Georgia in 1959 -comparison with the case of Typhoon Helen in 1958-. Kenkyu Jiho, 14, $621-630$ (in Japanese).

Wada, M., 1979: Numerical experiments of the tropical cyclone by use of the ArakawaSchubert parameterization. J. Meteor. Soc. Japan, 505-531.

Willoughby, H.E., H.-L. Jin, S.J. Lord and J.M. Piotrowicz, 1984 : Hurricane structure and evolution as simulated by an axisymmetric, nonhydrostatic numerical model. J. Atmos. Sci., 41, 1169-1186.
Yamasaki, M., 1968a : Numerical simulation of tropical cyclone development with the use of primitive equations. J. Meteor. Soc. Japan, 46, 178-201.

i

- $1968 \mathrm{~b}$ : Detailed analysis of a tropical cyclone simulated with a 13-layer model. Papers Meteor. Geophys., 19, 559-585.

- 1975 : A numerical experiment of the interaction between cumulus convection and larger-scale motion. Papers Meteor. Geophys., 26, 63-91.

— 1977a: A preliminary experiment of the tropical cyclone without parameterizing the effects of cumulus convection. $J$. Meteor. Soc. Japan, 55, 11-31.

- $1977 \mathrm{~b}$ : The role of surface friction in tropical cyclones. J. Meteor. Soc. Japan, 55, 559-572.

- 1979 : A further study of a CISK mode unaffected by surface friction. J. Meteor. Soc. Japan, 57, 112-132.

- 1982 : Typhoon. Tokyodo, 206 pp. (in Japanese).

— 1983: A further study of the tropical cyclone without parameterizing the effects of cumulus convection. Papers Meteor. Geophys., 34, 221-260.

- 1984 : Dynamics of convective clouds and "CISK" in vertical shear flow - with its application to easterly waves and squallline systems. J. Meteor. Soc. Japan, 62, 833863.

- , and H. Ohnishi, 1985 : A study of tropical cyclone motion related to asymmetric structure. Presented at the meeting of MSJ, Abstract No. 47, p. 37, (in Japanese).

Yanai, M., S. Esbensen and J. -H. Chu, 1973 : Determination of bulk properties of tropical cloud clusters from large-scale heat and moisture budgets. J. Atmos. Sci., $30,611-627$.

積雲対流をパラメタライズした台風 3 次元モデル

山岬 正紀

積雲対流をパラメタライズしないモデル（山岬，1977，1983 など）からの結果にもとづいて，従来のパラ 
メタリゼーションとは異なったモデルをつくり，それを台風 3 次元モデル(シグマ座標 5 層, 3 重格子, 最小 格子間隔 $20 \mathrm{~km})$ にくみこんで数值実験を行った。

従来の台風モデルでのスパイラルレインバンド（SRB）は重力波の性質をもっていたが，ここで得られた $\mathrm{SRB}$ は，パラメタライズしないモデルで得られたメソ対流の性質をもっている。すなわち, 雨の蒸発によっ て冷却した空気と，外からの暖かい湿った空気の相互作用が重要な役割を果している。メソ対流は多くの場 合 SRB の後端付近で発生して，下層風よりゆっくりと低気圧性に移動する。SRB や他の RB の構造，伝播 速度や方向, RB に対する地表摩擦の効果, RB を構成するメり対流の性質は, 台風の発達の時期, 強さ, RB の位置によって異なる。地表摩擦の効果を強くうけている SRB 内のメソ対流は，その外側からの空気の上昇 による対流要素の形成によって長時間維持される。台風強度になる前の時期でのメり対流の振舞いなどを含 めて, 得られた多くの結果は, パラメタライズしないモデルからの結果と一致している。

このような結果をもたらすパラメタリゼーションの重要な点および改善すべき点についても述べる。 